\title{
inu \\ Open-Circuit Fault-Tolerant Strategy for Interleaved Boost Converters via Filippov Method
}

\author{
Cristina Morel *, Ahmad Akrad, Rabia Sehab (D), Toufik Azib (D) and Cherif Larouci \\ Ecole Supérieure des Techniques Aéronautiques et de Construction Automobile, ESTACA'Lab Paris-Saclay, \\ 12 Avenue Paul Delouvrier, RD10, Montigny-le-Bretonneux, 78180 Paris, France; ahmad.akrad@estaca.fr (A.A.); \\ rabia.sehab@estaca.fr (R.S.); toufik.azib@estaca.fr (T.A.); cherif.larouci@estaca.fr (C.L.) \\ * Correspondence: cristina.morel@estaca.fr
}

check for updates

Citation: Morel, C.; Akrad, A.; Sehab, R.; Azib, T.; Larouci, C. Open-Circuit Fault-Tolerant Strategy for Interleaved Boost Converters via Filippov Method. Energies 2022, 15, 352. https://doi.org/10.3390/ en15010352

Academic Editor: Adolfo Dannier

Received: 2 December 2021

Accepted: 1 January 2022

Published: 4 January 2022

Publisher's Note: MDPI stays neutral with regard to jurisdictional claims in published maps and institutional affiliations.

Copyright: (C) 2022 by the authors. Licensee MDPI, Basel, Switzerland. This article is an open access article distributed under the terms and conditions of the Creative Commons Attribution (CC BY) license (https:// creativecommons.org/licenses/by/ $4.0 /)$.

\begin{abstract}
Interleaved converters use an increased number of power electronics switches; this may subsequently affect their reliability. However, this is an opportunity to develop fault-tolerant strategies to improve their reliability and to ensure continuity of service. This is why we herein propose, for the first time, a mathematical function to simultaneously model the healthy and faulty conditions of each switch, thus enabling a unique model of the system. This model is then used in an original fault-tolerant strategy based upon the peak current control with slope compensation. This method not only extends the stable range of the load variation but also ensures the stability in faulty conditions. Finally, the simulation results validate its effectiveness and confirm the theoretical analysis.
\end{abstract}

Keywords: fault-tolerant strategy; monodromy matrix; open-circuit; eigenvalues; slope compensation; interleaved converters

\section{Introduction}

Interleaved converters are applied to various applications as solar energy [1-4], fuel cell [5,6], power factor correction [7], automotive [8-10], and dc-dc switched-mode power converters [11], including wind systems [12,13]. The converters reliability is a great concern in such embedded applications. According to [14], the converters are sensitive to a failure of their semiconductor devices, which are their most fragile components. The system performance may be deteriorated by an open-circuit [5,15-20] or short-circuit [17] fault, which can be diagnosed through the inductive current measurement. Therefore, the converters with switch faults [4,21] are thoroughly studied.

Interleaving power converters allows to increase power density, voltage levels, and to improve EMC performances. The main advantages of interleaved $[5,16,20] \mathrm{dc}-\mathrm{dc}$ converters are the reduction of current and voltage ripples and the improvement of the high current control, compared to single-phase boost converters. Moreover, the passive components (inductor and capacitor) volume and mass are reduced. A computer-aided design methodology is introduced to design a power converter which determines the number of phases to optimize cost, size, and weight of the converter [10]. References [10,13,22,23] are limited to three, four, and five paralleled converters branches. Later, in 2004, Garcia analyzed an interleaved dc-dc converter with 16 and 32 phases [9].

In general, a dc-dc converter is a dynamical system with differential equations having discontinuous right-hand sides. The converter can exhibit unpredictable phenomena such as bifurcations and chaotic operations caused by the passage switching between several subsystems. The study of periodic orbit stability requires special techniques. The average technique $[24,25]$ is used to obtain the stability operation for the small switching period and to eliminate the nonlinear effects that occur on slow time scales. It destroys all the details about high clock frequency. Another widely used approach, which preserves the nonlinear effects, is the iterated map of the converter model. Reference [25] applies this technique and obtains a discrete observation at every clock's instant state, namely the Poincaré map. 
This nonlinear map is obtained with a local linearization at a fixed point. However, in many converters, such a map cannot be derived in a closed form because of the transcendental form of the involved equations.

In later work, the authors [26,27] used the Filippov approach as an alternative method to obtain the Jacobian of the Poincaré map. The Filippov method led to many papers in several kinds of mechanical and electrical applications, including systems with velocity reversals and viscoelastic supports [28,29], and buck, boost, and Cuk converters [26,27,30]. In the literature, this approach has also been applied to general piecewise dynamical systems [31-33]. The Filippov method describes the stability of periodic orbits by the monodromy matrix over a complete cycle [30,34]. This method treats each switching instant separately; the overall analysis is simpler than the conventional Poincaré map. The Filippov approach is much easier to obtain the eigenvalues of the monodromy matrix and to predict the converter stability. The conventional Poincaré map Jacobian approach and the Filippov method lead to the same results.

In the field of power electronics, the analysis of a single phase converter through Filippov method is now well established. In 2017, Wu [35] proposed the Filippov approach to investigate nonlinear phenomena of an interleaved boost converter. In this work, the authors developed a new technique to control the nonlinearity in an interleaved boost converter: the peak current control with slope compensation. Thus, with this control loop, the input voltage parameter range can be extended so that the 1T-period operation of the converter remains stable. A number of modifications of control strategies have been proposed, some aimed at increasing the accuracy of the stability and the range of the parameter variation. Earlier, another widely used supervising controller was introduced to study the system operation change from unstable periodic orbits to a periodic orbits. The Ott-Grebogi-Yorke approach [36] is a well-known chaos control method, for which no analytical knowledge of the system dynamics is required. Recently, delayed feedback control methods were developed by $[37,38]$ to stabilize the unstable periodic orbits. In the latter works, the authors develop other techniques such as as the filter-based noninvasive method, predictive control, frequency-domain, and Filippov's method with slope compensation approaches to impose a stable periodic orbit.

The influence of slope parameter cannot be investigated theoretically in most statespace-averaging-based methods: no relationship between the margin of stable operation and the slope parameter can be determined. The addition of a small-amplitude sinusoid to the ramp waveform is an alternative way to influence the saltation matrix, but it has very little influence on the switching instant, and hence on the normal operation of the converter. As the time derivative of the switching function contains a cosine term which attains maximum value at the switching instant, this term is affected. This significantly alters the saltation matrix and offers a handle in increasing the stability margin. In this approach, the compensation ramp directly affects the saltation matrix by influencing the time derivative of the switching function, and through that, the monodromy matrix. This matrix includes comprehensive information on the converter's parameters and the control loop. This method is an alternative to other existing methods. It also offers a deeper insight into the way the limit cycles lose their stability: it has the effect of pushing the eigenvalues inside the unit circle. From this analysis, an advanced control algorithm is developed to guarantee the satisfactory performance of the converter, avoiding nonlinear behaviors such as fast- and slow-scale bifurcations.

Our goal herein is to study the stability of a three-phase interleaved boost converter in healthy and open-circuit faulty conditions. This paper introduces a function which enables to distinguish between a defective open-circuit and an healthy open-circuit operation. First of all, the proposed function is universally applicable to any interleaved or single-phase dc-dc converters. More interesting, a single monodromy matrix is defined to understand and analyze the stability of the boost converters under different operating conditions, via Filippov's method. This matrix contains system information, including the converter parameters, the coefficients of the feedback loop controller, and the functions. In this paper, 
the Neimark bifurcation occurs where the complex conjugate eigenvalues are outside the unit circle equation, when the fixed point loses stability under healthy condition. To investigate such an event, it is necessary to compute the Floquet multiplier of the 1T-period orbit, which indicates the partial instability of the system with healthy conditions and a total instability with open-circuit fault for several loads and constant input voltage. Therefore, based on this premise, the same peak current control with slope compensation as above is used to ensure a fault-tolerance operation under the load variation of interleaved dc-dc converters and to increase the range of parameters (such as input voltage or load) over which the converter remains stable. This method is an alternative to other existing methods. It also offers a deeper insight into the way the limit cycles lose their stability: it has the effect of pushing the eigenvalues inside the unit circle. Moreover, we compare our theoretical results with the ones obtained by simulation.

The main aspects of this paper are as follows: a function to model the healthy and open-circuit conditions is introduced to model a three-phase interleaved boost converter in Section 2. In Section 3, this mathematical model (under different operating modes) is applied simultaneously to each switch, thus enabling a unique model of the system. Then, in Section 4, the saltation matrices and the monodromy matrix are detailed, followed by the identification of the duty cycle in Section 5, which allows the localization of the 1T-period limit cycle to predict the converter stability. Section 6 presents a control strategy with the slope compensation in order to increase the stability of the boost converter for several loads, and a constant input voltage. We end with a conclusion in Section 7.

\section{The Interleaved Boost Converter with Healthy and One Open-Circuit Faulty Condition}

In this paper, our study is focused on a three-phase interleaved boost converter, which consists of three independent boost branches. Let us consider the circuit of Figure 1 presenting its topology. We assume that the circuit parameters of each branch are exactly the same for the three levels connected in parallel with the current phase shifted by $\pi / 3$, as shown Figure 2 [35]. The parameters of the converter (the inductance, capacity, load resistance, clock frequency, input value, ...) are chosen to obtain the desired voltage ratio. The design of each branch satisfies the symmetry relation. Therefore, $L_{1}=L_{2}=L_{3}=L$ and $k_{L 1}=k_{L 2}=k_{L 3}=k_{L}$.

In addition, it is very important to have a control voltage loop [30] in order to keep the output voltage constant. The control strategies of the interleaved boost converter are voltage mode control and a peak current control. The circuit consists of inductances $L_{1}$, $L_{2}$, and $L_{3}$, diodes $D_{1}, D_{2}$, and $D_{3}$, switches $M O S_{1}, M O S_{2}$, and $M O S_{3}$, capacitance $C$, and the load $R$. $k_{i}$ and $k_{p}$ represent the gains of the PI controller. The feedback consists of a gain $k_{\mathcal{C}}$ applied to the output voltage $v_{\mathcal{C}}$ and a gain $k_{L}$ applied to the inductor currents $i_{L 1}$, $i_{L 2}$, and $i_{L 3}$. The output voltage $v_{\mathcal{C}}$ follows the reference signal $V_{\text {ref }}$ equation, the required output voltage. The converter itself is governed by five sets of linear differential equations related to the capacitor voltage $v_{\mathcal{C}}$, the inductor currents $i_{L 1}, i_{L 2}$, and $i_{L 3}$, and the output of the integrator in the feedback loop $v_{i}$. All these variables are considered as state variables of the system noted $x_{1}, x_{2}, x_{3}, x_{4}$, and $x_{5}$. 


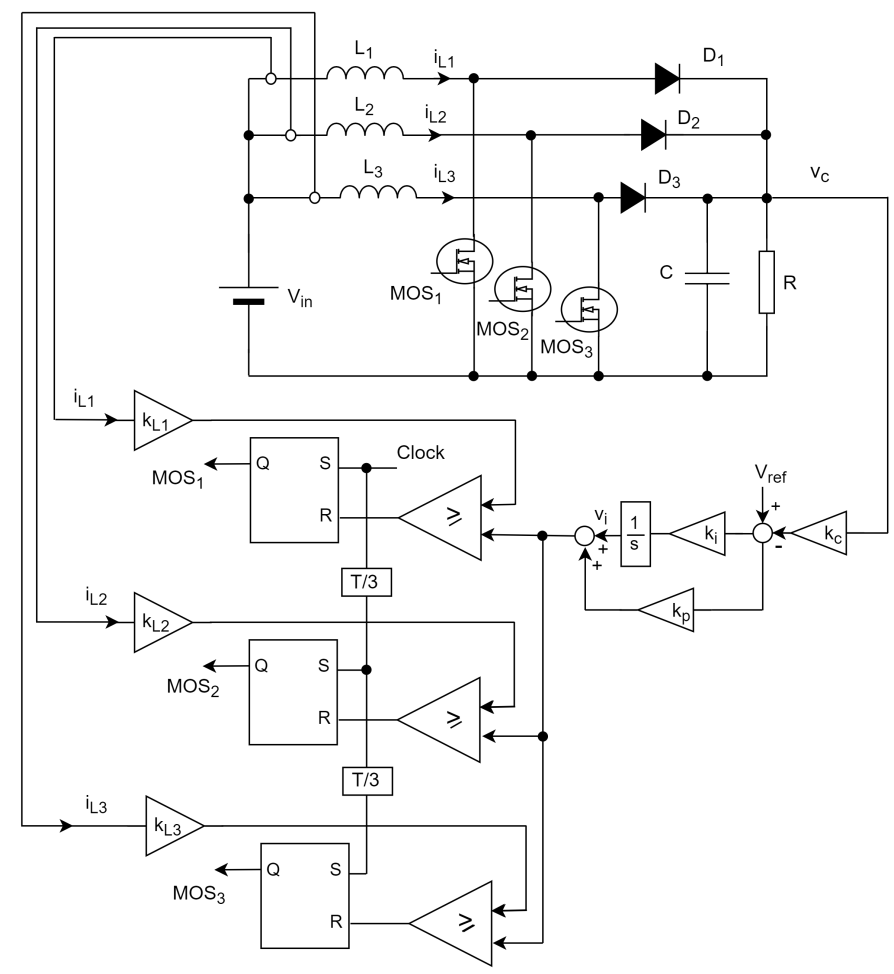

Figure 1. Interleaved boost converter with $V_{\text {in }}=14 \mathrm{~V}, V_{\text {ref }}=2.4 \mathrm{~V}, L_{1}=L_{2}=L_{3}=L=560 \mu \mathrm{H}$, $C=300 \mu \mathrm{F}, R=1.5 \Omega-2.5 \Omega, T=0.00001 \mathrm{~s}, k_{i}=50, k_{c}=0.1, k_{p}=0.5, k_{L 1}=k_{L 2}=k_{L 3}=k_{L}=0.1$.

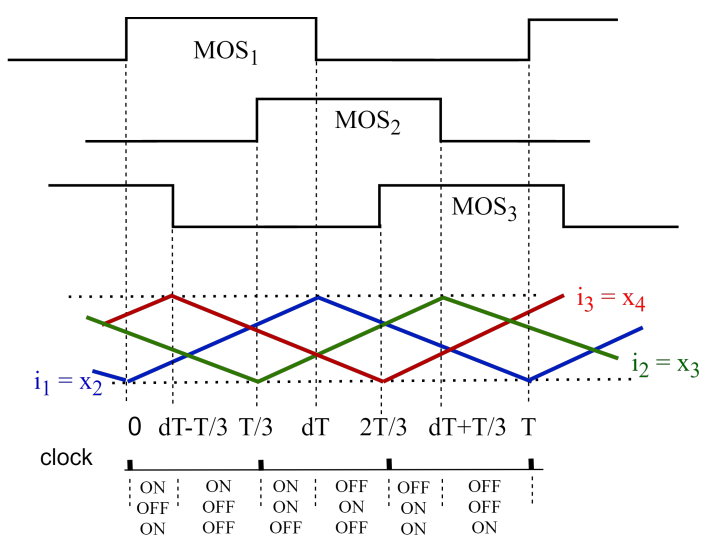

Figure 2. Gate pulses of $M O S_{1}, M O S_{2}$, and $M O S_{3}$, and the inductor currents $i_{L 1}, i_{L 2}$, and $i_{L 3}$ in steady state.

The branches are driven by gate pulses: the controllable devices $\left(\mathrm{MOS}_{1}, \mathrm{MOS}_{2}, \mathrm{MOS}_{3}\right)$ are switched at the same frequency with a $T / 3$ phase difference. Each branch has two modes, when the switch is either ON or OFF. Typical failures are open-circuit or shortcircuit of $\operatorname{MOS}_{i}(i=1,2,3)$ or no $R S$ latch trigger pulse output. We shall only consider the MOS open-circuit fault equation during the $O N$ mode.

In order to determine the mathematical model of the healthy and faulty conditions, a binary function $h_{i}(i=1,2,3)$ is defined as follows:

$$
h_{i}= \begin{cases}1 & \text { if the switching } \operatorname{MOS}_{i} \text { device is healthy } \\ 0 & \text { open-circuit fault of the } \operatorname{MOS}_{i} \text { device }(i=1,2,3) .\end{cases}
$$

Let us consider that an $M O S_{i}$ is turned $O N\left(\mathrm{i}=1\right.$ or 2 or 3 ) and the others, $M O S_{j}$ and $\operatorname{MOS}_{m}(j \neq i \neq m)$, are turned OFF. If the $M O S_{i}$ is healthy, then diode $D_{i}$ is reverse-biased and is OFF. Consequently, the input voltage (applied across the inductor $L_{i}$ ) becomes 
$u_{L i}=V_{i n}$. The currents through capacitance $C$ and load $R$ are provided by the inductor currents $i_{L j}$ and $i_{L m}: i_{C}+i_{R}=i_{L j}+i_{L m}(j \neq i \neq m)$. If an open-circuit fault of the $\operatorname{MOS}_{i}$ occurs when this one is turned $O N$, the voltage across $L_{i}$ is $u_{L i}=V_{\text {in }}-x_{1}$ ( $\mathrm{i}=1$ or 2 or $3)$. In this case, the currents through capacitance $C$ and load $R$ are $i_{C}+i_{R}=i_{L j}+i_{L i}+i_{L m}$ $(j \neq i \neq m)$. Consequently, a mathematical model is able to represent both the healthy and faulty conditions of the $M O S_{i}$ which can be defined by

$$
\begin{array}{ll}
V_{i n}=u_{L i}+\overline{h_{i}} \cdot x_{1} & i=1 \text { or } 2 \text { or } 3 ; \\
i_{C}+i_{R}=i_{L j}+\overline{h_{i}} \cdot i_{L i}+i_{L m}, & j \neq i \text { and } m \neq j .
\end{array}
$$

\section{Mathematical Model of the Boost Converter under Several Operating Modes}

The mathematical model [35] of the interleaved boost converter depends on the state of the switching devices $M O S_{1}, M O S_{2}$, and $M O S_{3}$. However, the converter evolves in six modes during a period, when the system is T-periodical.

\subsection{Mode ON OFF ON $[0 \leq t \leq d T-T / 3]$}

This mode occurs when $M O S_{1}$ is turned $\mathrm{ON}, M O S_{2}$ is in OFF-state, and $M O S_{3}$ is in $O N$-state. The current $i_{L 1}$ flows through $M O S_{1}$ and increases linearly from its initial value, as shown in Figure 2 . The current $i_{L 3}$ through $M O S_{3}$ also increases linearly and $i_{L 2}$, the current of $L_{2}$, flows through $D_{2}, C$, and $R$. In case of open-circuit faults of $M O S_{1}$ and $M O S_{3}$ too, the currents $i_{L 1}$ and $i_{L 3}$ are added to $i_{L 2}$. The capacitor and load currents are described by

$$
i_{C}+i_{R}=\overline{h_{1}} \cdot i_{L 1}+i_{L 2}+\overline{h_{3}} \cdot i_{L 3}, \quad \frac{d x_{1}}{d t}=-\frac{1}{R C} \cdot x_{1}+\frac{\overline{h_{1}}}{C} \cdot x_{2}+\frac{1}{C} \cdot x_{3}+\frac{\overline{h_{3}}}{C} \cdot x_{4} .
$$

The voltages across $L_{1}, L_{2}, L_{3}$ and the derivatives of $i_{L 1}, i_{L 2}, i_{L 3}$ can be expressed $[26,27]$ as follows:

$$
\begin{array}{rlrl}
V_{\text {in }} & =u_{L 1}+\overline{h_{1}} \cdot x_{1} & \frac{d x_{2}}{d t} & =-\frac{\overline{h_{1}}}{L} \cdot x_{1}+\frac{V_{\text {in }}}{L}, \\
V_{\text {in }} & =u_{L 2}+x_{1} & \frac{d x_{3}}{d t}=-\frac{1}{L} \cdot x_{1}+\frac{V_{\text {in }}}{L}, \\
V_{\text {in }}=u_{L 3}+\overline{h_{3}} \cdot x_{1} & \frac{d x_{4}}{d t}=-\frac{\overline{h_{3}}}{L} \cdot x_{1}+\frac{V_{\text {in }}}{L} .
\end{array}
$$

$v_{i}$, the control voltage of the integrator and its derivative $\frac{d x_{5}}{d t}$ are given by the following equations:

$$
v_{i}=\frac{k_{i}}{s}\left(V_{r e f}-k_{c} \cdot x_{1}\right), \quad \frac{d x_{5}}{d t}=-k_{i} k_{c} \cdot x_{1}+k_{i} \cdot V_{r e f} .
$$

The evolutions of the state variables in Mode ON OFF ON during $[0 \leq t \leq d T-T / 3]$ can be described by the following matrix equations and the right-side state equations as:

$$
A_{1}=\left[\begin{array}{ccccc}
-\frac{1}{R C} & \frac{h_{1}}{C} & \frac{1}{C} & \frac{h_{3}}{C} & 0 \\
-\frac{h_{1}}{L} & 0 & 0 & 0 & 0 \\
-\frac{1}{L} & 0 & 0 & 0 & 0 \\
-\frac{\bar{h}_{3}}{L} & 0 & 0 & 0 & 0 \\
-k_{i} k_{c} & 0 & 0 & 0 & 0
\end{array}\right], \quad B_{1}=\left[\begin{array}{cc}
0 & 0 \\
\frac{1}{L} & 0 \\
\frac{1}{L} & 0 \\
\frac{1}{L} & 0 \\
0 & k_{i}
\end{array}\right] . \quad f_{1}=\left[\begin{array}{c}
-\frac{1}{R C} x_{1}+\frac{\bar{h}_{1}}{C} x_{2}+\frac{1}{C} x_{3}+\frac{\bar{h}_{3}}{C} x_{4} \\
-\frac{\bar{h}_{1}}{L} \cdot x_{1}+\frac{1}{L} \cdot V_{i n} \\
-\frac{1}{L} \cdot x_{1}+\frac{1}{L} \cdot V_{i n} \\
-\frac{\bar{h}_{3}}{L} \cdot x_{1}+\frac{1}{L} \cdot V_{i n} \\
-k_{i} k_{c} \cdot x_{1}+k_{i} \cdot V_{i n}
\end{array}\right]
$$




\subsection{Mode ON OFF OFF $[d T-T / 3 \leq t \leq T / 3]$}

This mode occurs when $M S_{3}$ is turned OFF and when $i_{L 3}$ decreases linearly from the peak current according to the control strategy. $M O S_{1}$ is in $O N$-state, so the current $i_{L 1}$ through $M O S_{1}$ continues to increase linearly. $M O S_{2}$ is in OFF-state and $i_{L 2}$ decreases linearly until the occurrence of the clock pulse at $T / 3$. The capacitor and load currents are described by

$$
i_{C}+i_{R}=\overline{h_{1}} \cdot i_{L 1}+i_{L 2}+i_{L 3}, \quad \frac{d x_{1}}{d t}=-\frac{1}{R C} \cdot x_{1}+\frac{\overline{h_{1}}}{C} \cdot x_{2}+\frac{1}{C} \cdot x_{3}+\frac{1}{C} \cdot x_{4},
$$

The voltages across $L_{1}, L_{2}, L_{3}$ and the derivatives of $i_{L 1}, i_{L 2}, i_{L 3}$ can be calculated as follows:

$$
\begin{array}{rlrl}
V_{\text {in }} & =u_{L 1}+\overline{h_{1}} \cdot x_{1} & \frac{d x_{2}}{d t}=-\frac{\overline{h_{1}}}{L} \cdot x_{1}+\frac{V_{\text {in }}}{L}, \\
V_{\text {in }}=u_{L 2}+x_{1} & \frac{d x_{3}}{d t}=-\frac{1}{L} \cdot x_{1}+\frac{V_{\text {in }}}{L}, \\
V_{\text {in }}=u_{L 3}+x_{1} & \frac{d x_{4}}{d t}=-\frac{1}{L} \cdot x_{1}+\frac{V_{\text {in }}}{L} .
\end{array}
$$

$v_{i}$, the control voltage of the integrator, as well as $\frac{d x_{5}}{d t}$, are given in Equation (6). The evolution of the state variables during this interval can be described by the following matrix equations and the right-side state equations:

$$
A_{2}=\left[\begin{array}{ccccc}
-\frac{1}{R C} & \frac{\bar{h}_{1}}{C} & \frac{1}{C} & \frac{1}{C} & 0 \\
-\frac{\bar{h}_{1}}{L} & 0 & 0 & 0 & 0 \\
-\frac{1}{L} & 0 & 0 & 0 & 0 \\
-\frac{1}{L} & 0 & 0 & 0 & 0 \\
-k_{i} k_{c} & 0 & 0 & 0 & 0
\end{array}\right], \quad B_{2}=B_{1}, \quad f_{2}=\left[\begin{array}{c}
-\frac{1}{R C} x_{1}+\frac{\bar{h}_{1}}{C} x_{2}+\frac{1}{C} x_{3}+\frac{1}{C} x_{4} \\
-\frac{\bar{h}_{1}}{L} \cdot x_{1}+\frac{1}{L} \cdot V_{i n} \\
-\frac{1}{L} \cdot x_{1}+\frac{1}{L} \cdot V_{i n} \\
-\frac{1}{L} \cdot x_{1}+\frac{1}{L} \cdot V_{i n} \\
-k_{i} k_{c} \cdot x_{1}+k_{i} \cdot V_{i n}
\end{array}\right] .
$$

\subsection{Mode ON ON OFF $[T / 3 \leq t \leq d T]$}

$M O S_{2}$ is turned ON due to the impulse clock, MOS 1 is in $O N$-state, and $M O S_{3}$ is in OFF-state. The current $i_{L 1}$ through $M O S_{1}$ continues to increase linearly until the peak current, according to the control strategy. In addition, $i_{L 2}$ increases linearly and $i_{L 3}$ also continues to decrease. Similarly, the capacitor current and the voltages across $L_{1}, L_{2}, L_{3}$ are described by

$$
i_{C}+i_{R}=\overline{h_{1}} \cdot i_{L 1}+i_{L 2}+i_{L 3}, \quad \frac{d x_{1}}{d t}=-\frac{1}{R C} \cdot x_{1}+\frac{\overline{h_{1}}}{C} \cdot x_{2}+\frac{1}{C} \cdot x_{3}+\frac{1}{C} \cdot x_{4},
$$

and

$$
\begin{array}{ll}
V_{\text {in }}=u_{L 1}+\overline{h_{1}} \cdot x_{1} & \frac{d x_{2}}{d t}=-\frac{\overline{h_{1}}}{L} \cdot x_{1}+\frac{V_{\text {in }}}{L}, \\
V_{\text {in }}=u_{L 2}+x_{1} & \frac{d x_{3}}{d t}=-\frac{1}{L} \cdot x_{1}+\frac{V_{i n}}{L}, \\
V_{\text {in }}=u_{L 3}+\overline{h_{3}} \cdot x_{1} & \frac{d x_{4}}{d t}=-\frac{\overline{h_{3}}}{L} \cdot x_{1}+\frac{V_{\text {in }}}{L} .
\end{array}
$$


Equation (6) is still available to define the control voltage of the integrator $v_{i}$ and its derivative. The evolution of the state variables in Mode ON OFF OFF during $[T / 3 \leq t \leq d T]$ can be described as

$$
A_{3}=\left[\begin{array}{ccccc}
-\frac{1}{R C} & \frac{h_{1}}{C} & \frac{h_{2}}{C} & \frac{1}{C} & 0 \\
-\frac{h_{1}}{L} & 0 & 0 & 0 & 0 \\
-\frac{h_{2}}{L} & 0 & 0 & 0 & 0 \\
-\frac{1}{L} & 0 & 0 & 0 & 0 \\
-k_{i} k_{c} & 0 & 0 & 0 & 0
\end{array}\right], \quad B_{3}=B_{1}, \quad f_{3}=\left[\begin{array}{c}
-\frac{1}{R C} x_{1}+\frac{\bar{h}_{1}}{C} x_{2}+\frac{\bar{h}_{2}}{C} x_{3}+\frac{1}{C} x_{4} \\
-\frac{\bar{h}_{1}}{L} \cdot x_{1}+\frac{1}{L} \cdot V_{i n} \\
-\frac{\bar{h}_{2}}{L} \cdot x_{1}+\frac{1}{L} \cdot V_{i n} \\
-\frac{1}{L} \cdot x_{1}+\frac{1}{L} \cdot V_{i n} \\
-k_{i} k_{c} \cdot x_{1}+k_{i} \cdot V_{i n}
\end{array}\right] .
$$

3.4. Mode OFF ON OFF $[d T \leq t \leq 2 T / 3]$

$M O S_{1}$ is turned OFF, as $M O S_{2}$ is in $O N$-state and $M O S_{3}$ is in OFF-state. The current $i_{L 1}$ through $M O S_{1}$ decreases linearly from peak current, according to the control strategy. The current $i_{L 2}$ continues to increase linearly, and the current $i_{L 3}$ continues to decrease linearly. Similarly, the capacitor and load current are described by

$$
i_{C}+i_{R}=i_{L 1}+\overline{h_{2}} \cdot i_{L 2}+i_{L 3}, \quad \frac{d x_{1}}{d t}=-\frac{1}{R C} \cdot x_{1}+\frac{1}{C} \cdot x_{2}+\frac{\overline{h_{2}}}{C} \cdot x_{3}+\frac{1}{C} \cdot x_{4} .
$$

The voltages across $L_{1}, L_{2}, L_{3}$ and the derivatives of $i_{L 1}, i_{L 2}, i_{L 3}$ can be expressed as follows:

$$
\begin{array}{rlrl}
V_{\text {in }} & =u_{L 1}+x_{1} & \frac{d x_{2}}{d t}=-\frac{1}{L} \cdot x_{1}+\frac{V_{\text {in }}}{L}, \\
V_{\text {in }}=u_{L 2}+\overline{h_{2}} \cdot x_{1} & \frac{d x_{3}}{d t}=-\frac{\overline{h_{2}}}{L} \cdot x_{1}+\frac{V_{\text {in }}}{L}, \\
V_{\text {in }}=u_{L 3}+x_{1} & \frac{d x_{4}}{d t}=-\frac{1}{L} \cdot x_{1}+\frac{V_{\text {in }}}{L} .
\end{array}
$$

The control voltage $v_{i}$ obtained from the output of the integrator and its derivative $\frac{d x_{5}}{d t}$ are as in Equation (6). During $[d T \leq t \leq 2 T / 3]$ intervals, the matrix form of the state equations are

$$
A_{4}=\left[\begin{array}{ccccc}
-\frac{1}{R C} & \frac{1}{C} & \frac{\bar{h}_{2}}{C} & \frac{1}{C} & 0 \\
-\frac{1}{L} & 0 & 0 & 0 & 0 \\
-\frac{\bar{h}_{2}}{L} & 0 & 0 & 0 & 0 \\
-\frac{1}{L} & 0 & 0 & 0 & 0 \\
-k_{i} k_{c} & 0 & 0 & 0 & 0
\end{array}\right], \quad B_{4}=B_{1}, \quad f_{4}=\left[\begin{array}{c}
-\frac{1}{R C} x_{1}+\frac{1}{C} x_{2}+\frac{\bar{h}_{2}}{C} x_{3}+\frac{1}{C} x_{4} \\
-\frac{1}{L} \cdot x_{1}+\frac{1}{L} \cdot V_{i n} \\
-\frac{\bar{h}_{2}}{L} \cdot x_{1}+\frac{1}{L} \cdot V_{i n} \\
-\frac{1}{L} \cdot x_{1}+\frac{1}{L} \cdot V_{i n} \\
-k_{i} k_{c} \cdot x_{1}+k_{i} \cdot V_{i n}
\end{array}\right] .
$$

\subsection{Mode OFF ON ON $[2 T / 3 \leq t \leq d T+T / 3]$}

$M O S_{1}$ is in OFF-state, $M O S_{2}$ is in $O N$-stat,e and $M_{3} S_{3}$ is turned to $O N$-state. The current $i_{L 1}$ through $M O S_{1}$ continues to decrease linearly. The current $i_{L 2}$ increases linearly until the peak current, according to the control strategy. The current $i_{L 3}$ begins to increase after the pulse clock at $2 T / 3$ time. Similarly, the capacitor and load current are described by 


$$
\begin{aligned}
& i_{C}+i_{R}=i_{L 1}+\overline{h_{2}} \cdot i_{L 2}+\overline{h_{3}} \cdot i_{L 3}, \quad \frac{d x_{1}}{d t}=-\frac{1}{R C} \cdot x_{1}+\frac{1}{C} \cdot x_{2}+\frac{\overline{h_{2}}}{C} \cdot x_{3}+\frac{\overline{h_{3}}}{C} \cdot x_{4} . \\
& V_{\text {in }}=u_{L 1}+x_{1} \quad \frac{d x_{2}}{d t}=-\frac{1}{L} \cdot x_{1}+\frac{V_{\text {in }}}{L}, \\
& V_{\text {in }}=u_{L 2}+\overline{h_{2}} \cdot x_{1} \quad \frac{d x_{3}}{d t}=-\frac{\overline{h_{2}}}{L} \cdot x_{1}+\frac{V_{\text {in }}}{L}, \\
& V_{\text {in }}=u_{L 3}+\overline{h_{3}} \cdot x_{1} \quad \frac{d x_{4}}{d t}=-\frac{\overline{h_{3}}}{L} \cdot x_{1}+\frac{V_{\text {in }}}{L} . \\
& A_{5}=\left[\begin{array}{ccccc}
-\frac{1}{R C} & \frac{1}{C} & \frac{\bar{h}_{2}}{C} & \frac{\bar{h}_{3}}{C} & 0 \\
-\frac{1}{L} & 0 & 0 & 0 & 0 \\
-\frac{\bar{h}_{2}}{L} & 0 & 0 & 0 & 0 \\
-\frac{\bar{h}_{3}}{L} & 0 & 0 & 0 & 0 \\
-k_{i} k_{c} & 0 & 0 & 0 & 0
\end{array}\right], \quad B_{5}=B_{1}, \quad f_{5}=\left[\begin{array}{c}
-\frac{1}{R C} x_{1}+\frac{1}{C} x_{2}+\frac{\bar{h}_{2}}{C} x_{3}+\frac{\bar{h}_{3}}{C} x_{4} \\
-\frac{1}{L} \cdot x_{1}+\frac{1}{L} \cdot V_{i n} \\
-\frac{\bar{h}_{2}}{L} \cdot x_{1}+\frac{1}{L} \cdot V_{i n} \\
-\frac{\bar{h}_{3}}{L} \cdot x_{1}+\frac{1}{L} \cdot V_{i n} \\
-k_{i} k_{c} \cdot x_{1}+k_{i} \cdot V_{i n}
\end{array}\right] .
\end{aligned}
$$

\subsection{Mode OFF OFF ON $[d T+T / 3 \leq t \leq T]$}

For this last configuration, $M O S_{1}$ is in OFF-state, $M O S_{2}$ is turned $O F F$, and $M O S_{3}$ is in $O N$-state. The current $i_{L 1}$ through $M O S_{1}$ continues to decrease linearly until the next pulse clock arrives. The current $i_{L 2}$ begins to decrease linearly, and the current $i_{L 3}$ continues to increase linearly. Similarly, the capacitor and load current are described by

$$
i_{C}+i_{R}=i_{L 1}+i_{L 2}+\overline{h_{3}} \cdot i_{L 3}, \quad \frac{d x_{1}}{d t}=-\frac{1}{R C} \cdot x_{1}+\frac{1}{C} \cdot x_{2}+\frac{1}{C} \cdot x_{3}+\frac{\overline{h_{3}}}{C} \cdot x_{4} .
$$

The voltages across $L_{1}, L_{2}, L_{3}$ and the derivatives of $i_{L 1}, i_{L 2}, i_{L 3}$ are expressed as follows:

$$
\begin{aligned}
V_{\text {in }} & =u_{L 1}+x_{1} & \frac{d x_{2}}{d t} & =-\frac{1}{L} \cdot x_{1}+\frac{V_{\text {in }}}{L}, \\
V_{\text {in }} & =u_{L 2}+x_{1} & \frac{d x_{3}}{d t} & =-\frac{1}{L} \cdot x_{1}+\frac{V_{\text {in }}}{L}, \\
V_{\text {in }} & =u_{L 3}+\overline{h_{3}} \cdot x_{1} & \frac{d x_{4}}{d t} & =-\frac{\bar{h}}{L} \cdot x_{1}+\frac{V_{\text {in }}}{L} .
\end{aligned}
$$

$v_{i}$, the control voltage of the integrator, and its derivative $\frac{d x_{5}}{d t}$ are given by Equation (6). The evolution of the state variables in Mode OFF OFF ON during $[d T+T / 3 \leq t \leq T]$ are described by the following matrix equations and the right-hand side state equations: 


$$
A_{6}=\left[\begin{array}{ccccc}
-\frac{1}{R C} & \frac{1}{C} & \frac{1}{C} & \frac{h_{3}}{C} & 0 \\
-\frac{1}{L} & 0 & 0 & 0 & 0 \\
-\frac{1}{L} & 0 & 0 & 0 & 0 \\
-\frac{\bar{h}_{3}}{L} & 0 & 0 & 0 & 0 \\
-k_{i} k_{c} & 0 & 0 & 0 & 0
\end{array}\right], \quad B_{6}=B_{1}, \quad f_{6}=\left[\begin{array}{c}
-\frac{1}{R C} x_{1}+\frac{1}{C} x_{2}+\frac{1}{C} x_{3}+\frac{\bar{h}_{3}}{C} x_{4} \\
-\frac{1}{L} \cdot x_{1}+\frac{1}{L} \cdot V_{i n} \\
-\frac{1}{L} \cdot x_{1}+\frac{1}{L} \cdot V_{i n} \\
-\frac{\bar{h}_{3}}{L} \cdot x_{1}+\frac{1}{L} \cdot V_{i n} \\
-k_{i} k_{c} \cdot x_{1}+k_{i} \cdot V_{i n}
\end{array}\right] .
$$

\section{Saltation and Monodromy Matrices for the Interleaved Boost Converter}

A linear time-invariant system is represented by the differential equation $\dot{x}(t)=f(x)$, with initial conditions. An interleaved boost converter is a the discontinuous dynamical system composed of six smooth vector fields that are separated by discontinuity boundaries. This nonlinear system, with a discontinuous right-hand side, is described by the following differential equations:

$$
\dot{x}=f(t, x(t))= \begin{cases}f_{1}(t, x(t)), & x \in V_{1} \\ f_{2}(t, x(t)), & x \in V_{2} \\ f_{3}(t, x(t)), & x \in V_{3} \\ f_{4}(t, x(t)), & x \in V_{4} \\ f_{5}(t, x(t)), & x \in V_{5} \\ f_{6}(t, x(t)), & x \in V_{6}\end{cases}
$$

where $f_{1}$ of Equation (7), $f_{2}$ of Equation (10), $f_{3}$ of Equation (13), $f_{4}$ of Equation (16), $f_{5}$ of Equation (19), and $f_{6}$ of Equation (22) are six smooth functions, and $x \in \mathbb{R}^{5}$. For simplicity reasons, let us consider a region of the state space $\mathbb{R}^{5}$ which splits into six subspaces [30,34]: $V_{1}$ and $V_{2}$ are separated by surface $\Sigma_{1}, V_{3}$ and $V_{4}$ are separated by surface $\Sigma_{2}$, and $V_{5}$ and $V_{6}$ are separated by surface $\Sigma_{3}$, denoted by Figure 3, with:

$$
\begin{array}{lll}
V_{1}=\left\{x \in \mathbb{R}^{5}: s w_{1}(x(t))<0\right\} & V_{2}=\left\{x \in \mathbb{R}^{5}: s w_{1}(x(t))>0\right\} & \Sigma_{1}=\left\{x \in \mathbb{R}^{5}: s w_{1}(t, x)=0\right\}, \\
V_{3}=\left\{x \in \mathbb{R}^{5}: s w_{2}(x(t))<0\right\} & V_{4}=\left\{x \in \mathbb{R}^{5}: s w_{2}(x(t))>0\right\} & \Sigma_{2}=\left\{x \in \mathbb{R}^{5}: s w_{2}(t, x)=0\right\}, \\
V_{5}=\left\{x \in \mathbb{R}^{5}: s w_{3}(x(t))<0\right\} & V_{6}=\left\{x \in \mathbb{R}^{5}: s w_{3}(x(t))>0\right\} & \Sigma_{3}=\left\{x \in \mathbb{R}^{5}: s w_{3}(t, x)=0\right\},
\end{array}
$$

where $s w_{1}(x(t)), s w_{2}(x(t))$, and $s w_{3}(x(t))$ are scalar switching functions. It is presupposed that $f_{i}(i=\overline{1,6})$ is time continuous and discontinuous in $x_{i}(i=\overline{1,5})$, and crosses $\Sigma_{r}(r=\overline{1,3})$ transversally. Discontinuous systems exhibit jumps in the evolution of the fundamental solution matrix. Filippov showed that the system of Equation (23) can be transformed into a system continuous at the switching instant [29]. Therefore, the discontinuous problem can be replaced by a continuous set-valued one as follows:

$$
\dot{x}(t)=F(t, x(t))= \begin{cases}f_{i}(t, x(t)) & \text { if } x \in V_{i}, \\ q f_{i}(t, x(t))+(1-q) f_{i+1}(t, x(t)) & \text { if } x \in \Sigma_{r}, \\ f_{i+1}(t, x(t)) & \text { if } x \in V_{i+1} .\end{cases}
$$

where $q \in[0,1], i=\overline{1,5}$, and $r=\overline{1,3}$. Now, on $\Sigma_{r}(r=\overline{1,3})$, the discontinuity is replaced with the segment, which means that on $\Sigma_{r}(\mathrm{r}=\overline{1,3}), F$ consists of infinite number of points as shown in Figure 4. $x\left(t_{i}\right)$ passing from the subsystem is described by the vector $f_{i}$ (Equation, using the fundamental solution matrix just before the jump $t_{\Sigma_{-}}$) intersects the 
switching surface, and goes to the subsystem described by the vector $f_{i+1}$ (Equation, using the fundamental solution matrix just after the jump $t_{\Sigma_{+}}$).

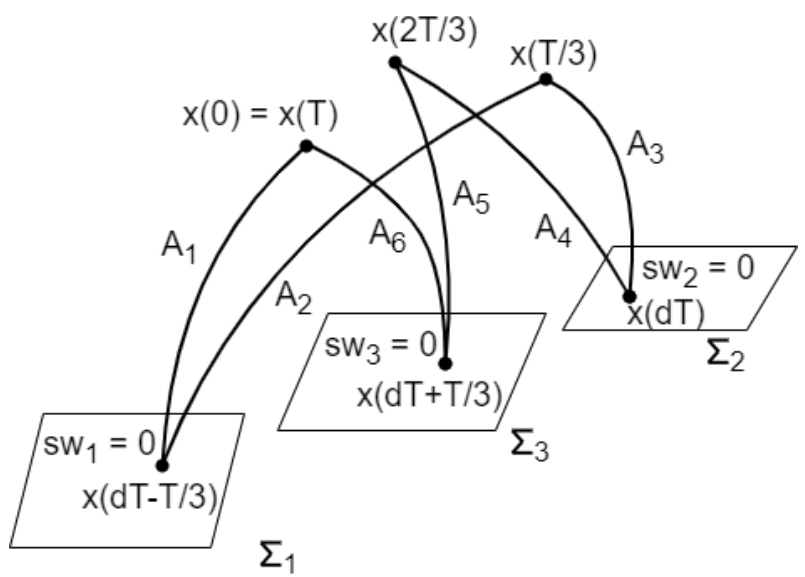

Figure 3. One cycle of orbit evolution of state variables $x(t)$.

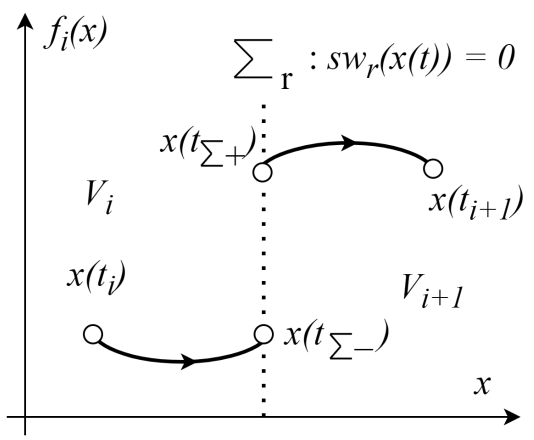

a)

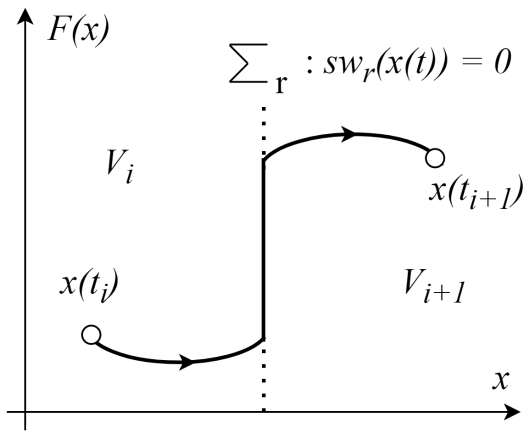

b)

Figure 4. (a) Graph of the function $f(x) ;(\mathbf{b}) F(x)$ at $t_{0}$ goes over the instant $t_{\Sigma}$ with a smooth jump up to the instant $T$.

The transition at each switching moment is described by saltation matrices which can be represented as follows:

$$
S_{i i+1}=\mathbb{I}_{5}+\frac{\left(f_{i+1}-f_{i}\right) n^{T}}{n_{i}^{T} f_{i}+\frac{\partial s w_{r}}{\partial t}}
$$

where $f_{i}$ and $f_{i+1}, i=\overline{1,5}$ are the right-hand sides of the state equations, before and after switching, where $n$ is a normal vector to the switching surface and $n=\nabla s w_{r}(x(t))$.

As mentioned above, we are interested in the stability of the dynamical system, Equation (23), where the system regains a T-periodic orbit. One method to check the stability of periodic motions is to calculate, over one complete cycle $[0, T)$, the state transition matrix (monodromy matrix $\Phi_{\text {cycle } 1}$ of Figure 5) of the system and then to determine its eigenvalues. If they are inside of the unit circle, then the periodic motion is stable. For an interleaved boost converter, the state evolves through six subsystems: ON and OFF states of the $M O S_{1}$, $M O S_{2}$, and $M O S_{3}$. As we are interested in the stability of the periodic orbit exhibited by the converters, we need to calculate the state transition matrix over a whole clock cycle. The monodromy matrix must be broken into six state transition matrices of the regions before and after the switching, and five for the transition across the switching surfaces. 


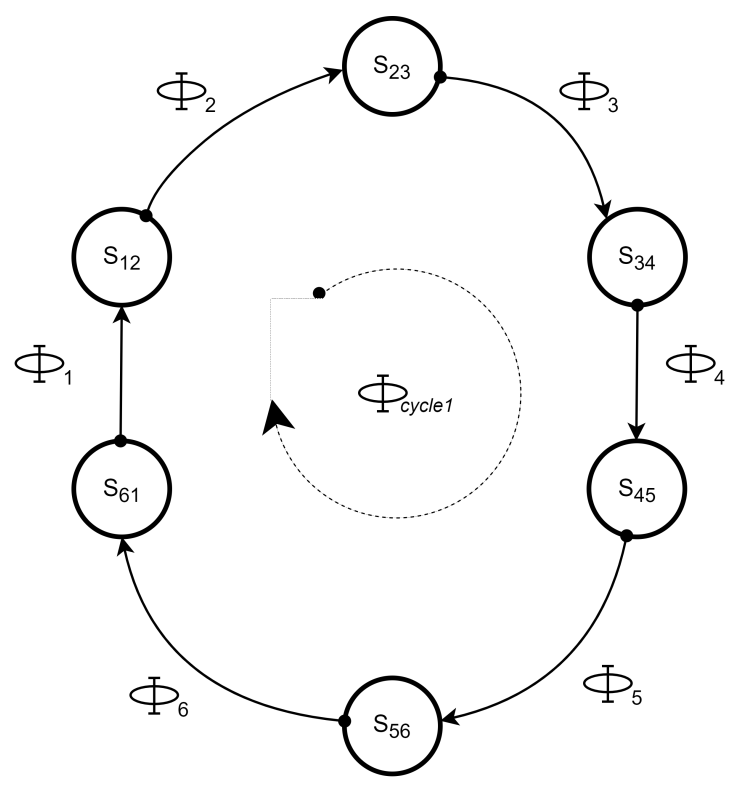

Figure 5. Diagram of the transition matrices and the saltation matrices in healthy switching case $h_{1}=h_{2}=h_{3}=1$.

This matrix $\Phi_{\text {cycle } 1}$ is composed of the fundamental solution matrices where the vector field is smooth and of the saltation matrices where the vector field crosses a switching boundary $\Sigma_{r}$ :

$$
\begin{aligned}
\Phi_{\text {cycle } 1}(T, 0)= & S_{61} \cdot \Phi_{6}\left(T, d T+\frac{T}{3}\right) \cdot S_{56} \cdot \Phi_{5}\left(d T+\frac{T}{3}, \frac{2 T}{3}\right) \cdot S_{45} \cdot \Phi_{4}\left(\frac{2 T}{3}, d T\right) . \\
& \cdot S_{34} \cdot \Phi_{3}\left(d T, \frac{T}{3}\right) \cdot S_{23} \cdot \Phi_{2}\left(\frac{T}{3}, d T-\frac{T}{3}\right) \cdot S_{12} \cdot \Phi_{1}\left(d T-\frac{T}{3}, 0\right)
\end{aligned}
$$

and

$$
\Phi_{c y c l e 1}(T, 0)=e^{A_{6}\left(\frac{2 T}{3}-d T\right)} \cdot S_{56} \cdot e^{A_{5}\left(d T-\frac{T}{3}\right)} \cdot e^{A_{4}\left(\frac{2 T}{3}-d T\right)} \cdot c d o t S_{34} \cdot e^{A_{3}\left(d T-\frac{T}{3}\right)} \cdot e^{A_{2}\left(\frac{2 T}{3}-d T\right)} \cdot S_{12} \cdot e^{A_{1}\left(d T-\frac{T}{3}\right)} .
$$

$\Phi_{6}, \Phi_{5}, \Phi_{4}, \Phi_{3}, \Phi_{2}$, and $\Phi_{1}$ are fundamental solution matrices depending on $A_{6}, A_{5}, A_{4}$, $A_{3}, A_{2}$ and $A_{1} . S_{12}, S_{23}, S_{34}, S_{45}, S_{56}$, and $S_{61}$ are the saltation matrices calculated at the instants $d T-T / 3, T / 3, d T, 2 T / 3, T / 3+d T$, and $T$. The saltation matrices $S_{23}, S_{45}$, and $S_{61}$ are related to the switching from $O F F$ to $O N$ modes. These matrices turn out to identical matrices $\mathbb{I}_{5}$, because $s w_{r}(r=\overline{1,3})$ are discontinuous at the instants $(T / 3,2 T / 3$, and $T)$, and in consequence $\partial s w_{r} / \partial t=$ infinite.

From the circuit topology (Figure 1), the switching functions are

$$
\begin{aligned}
& s w_{1}(x, t)=k_{L} \cdot x_{4}(t)-x_{5}(t)+k_{p} \cdot\left(k_{c} \cdot x_{1}(t)-V_{r e f}\right), \\
& s w_{2}(x, t)=k_{L} \cdot x_{2}(t)-x_{5}(t)+k_{p} \cdot\left(k_{c} \cdot x_{1}(t)-V_{r e f}\right), \\
& s w_{3}(x, t)=k_{L} \cdot x_{3}(t)-x_{5}(t)+k_{p} \cdot\left(k_{c} \cdot x_{1}(t)-V_{r e f}\right),
\end{aligned}
$$

and their time derivation

$$
\frac{\partial s w_{1}}{\partial t}=\frac{\partial s w_{2}}{\partial t}=\frac{\partial s w_{3}}{\partial t}=0 .
$$

The normal vectors at the point $x$ are given by 


$$
\begin{gathered}
\frac{\partial s w_{1}}{\partial x}=n_{1}=\left[\begin{array}{c}
\frac{\partial s w_{1}}{\partial x_{1}} \\
\frac{\partial s w_{1}}{\partial x_{2}} \\
\frac{\partial s w_{1}}{\partial x_{3}} \\
\frac{\partial s w_{1}}{\partial x_{4}} \\
\frac{\partial s w_{1}}{\partial x_{5}}
\end{array}\right]=\left[\begin{array}{c}
k_{p} k_{c} \\
0 \\
0 \\
k_{L} \\
-1
\end{array}\right], \\
\frac{\partial s w_{2}}{\partial x}=n_{2}=\left[\begin{array}{c}
\frac{\partial s w_{2}}{\partial x_{1}} \\
\frac{\partial s w_{2}}{\partial x_{2}} \\
\frac{\partial s w_{2}}{\partial x_{3}} \\
\frac{\partial s w_{2}}{\partial x_{4}} \\
\frac{\partial w_{2}}{\partial x_{5}}
\end{array}\right]=\left[\begin{array}{c}
k_{p} k_{c} \\
k_{L} \\
0 \\
0 \\
-1
\end{array}\right]
\end{gathered}
$$

and

$$
\frac{\partial s w_{3}}{\partial x}=n_{3}=\left[\begin{array}{c}
\frac{\partial s w_{3}}{\partial x_{1}} \\
\frac{\partial s w_{3}}{\partial x_{2}} \\
\frac{\partial s w_{3}}{\partial x_{3}} \\
\frac{\partial s w_{3}}{\partial x_{4}} \\
\frac{\partial s w_{3}}{\partial x_{5}}
\end{array}\right]=\left[\begin{array}{c}
k_{p} k_{c} \\
0 \\
k_{L} \\
0 \\
-1
\end{array}\right] .
$$

By substitution of Equations (7), (10), (30), and (31) into Equation (26), the saltation matrix $S_{12}$ at the moment $t=d T-\frac{T}{3}$ can be obtained in the following form:

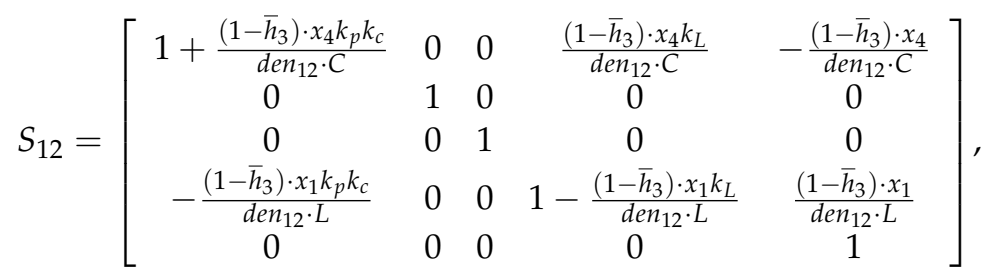

where

$$
\operatorname{den}_{12}=\left(k_{i} k_{c}-\frac{k_{p} k_{c}}{R C}-\frac{k_{L} \bar{h}_{3}}{L}\right) \cdot x_{1}+k_{p} k_{c} \frac{\bar{h}_{1}}{C} \cdot x_{2}+\frac{k_{p} k_{c}}{C} \cdot x_{3}+k_{p} k_{c} \frac{\bar{h}_{3}}{C} \cdot x_{4}+\frac{k_{L}}{L} \cdot V_{\text {in }}-k_{i} \cdot V_{\text {ref }} \text {. }
$$

With Equations (13), (16), (30), (32), and (26), at $t=d T$, the saltation matrix $S_{34}$ is given by

$$
S_{34}=\left[\begin{array}{ccccc}
1+\frac{\left(1-\bar{h}_{1}\right) \cdot x_{2} k_{p} k_{c}}{d e_{34} \cdot C} & \frac{\left(1-\bar{h}_{1}\right) \cdot x_{2} k_{L}}{d e n_{34} \cdot C} & 0 & 0 & -\frac{\left(1-\bar{h}_{1}\right) \cdot x_{2}}{d e n_{34} \cdot C} \\
-\frac{\left(1-\bar{h}_{1}\right) \cdot x_{1} k_{p} k_{c}}{d e_{34} \cdot L} & 1-\frac{\left(1-h_{1}\right) \cdot x_{1} k_{L}}{d n_{34} \cdot L} & 0 & 0 & \frac{\left(1-\bar{n}_{1}\right) \cdot x_{1}}{d e n_{34} \cdot L} \\
0 & 0 & 1 & 0 & 0 \\
0 & 0 & 0 & 1 & 0 \\
0 & 0 & 0 & 0 & 1
\end{array}\right],
$$

where

$$
\operatorname{den}_{34}=\left(k_{i} k_{c}-\frac{k_{p} k_{c}}{R C}-\frac{k_{L} \bar{h}_{1}}{L}\right) \cdot x_{1}+k_{p} k_{c} \frac{\bar{h}_{1}}{C} \cdot x_{2}+k_{p} k_{c} \frac{\bar{h}_{2}}{C} \cdot x_{3}+\frac{k_{p} k_{c}}{C} \cdot x_{4}+\frac{k_{L}}{L} \cdot V_{i n}-k_{i} \cdot V_{r e f} \text {. }
$$

Using Equations (19), (22), (30), (33), and (26), the saltation matrix $S_{56}$ at the instant $t=d T+\frac{T}{3}$ becomes 


$$
S_{56}=\left[\begin{array}{ccccc}
1+\frac{\left(1-\bar{h}_{2}\right) \cdot x_{3} k_{p} k_{c}}{\operatorname{den}_{56} \cdot C} & 0 & \frac{\left(1-\bar{h}_{2}\right) \cdot x_{3} k_{L}}{\operatorname{den}_{56} \cdot C} & 0 & -\frac{\left(1-\bar{h}_{2}\right) \cdot x_{3}}{\operatorname{den} n_{56} \cdot C} \\
0 & 1 & 0 & 0 & 0 \\
-\frac{\left(1-\bar{h}_{2}\right) \cdot x_{1} k_{p} k_{c}}{\operatorname{den}_{56} \cdot L} & 0 & 1-\frac{\left(1-\bar{h}_{2}\right) \cdot x_{1} k_{L}}{\operatorname{den} n_{6} \cdot L} & 0 & \frac{\left(1-\bar{h}_{2}\right) \cdot x_{1}}{\operatorname{den} 56 \cdot L} \\
0 & 0 & 0 & 1 & 0 \\
0 & 0 & 0 & 0 & 1
\end{array}\right]
$$

where

$$
\operatorname{den}_{56}=\left(k_{i} k_{c}-\frac{k_{p} k_{c}}{R C}-\frac{k_{L} \bar{h}_{2}}{L}\right) \cdot x_{1}+\frac{k_{p} k_{c}}{C} \cdot x_{2}+k_{p} k_{c} \frac{\bar{h}_{2}}{C} \cdot x_{3}+k_{p} k_{c} \frac{\bar{h}_{3}}{C} \cdot x_{4}+\frac{k_{L}}{L} \cdot V_{i n}-k_{i} \cdot V_{r e f}
$$

\section{Identification of Duty Cycles of the Period-1T Limit Cycle}

The duty cycle $d$ is calculated in order to locate the limit cycle for several values of the load $R$. When the switch occurs on the surface $\Sigma_{1}$, the state vector at $t=d T-\frac{T}{3}$ is given by

$$
x(d T-T / 3)=e^{A_{1} \cdot(d T-T / 3)} \cdot x(0)+\int_{0}^{d T-T / 3} e^{A_{1} \cdot(d T-T / 3-\tau)} \cdot B_{1} u d \tau=\Phi_{1} \cdot x(0)+\text { Integ }_{1} .
$$

The state vector at the clock pulse $t=T / 3$ is as follows:

$$
x(T / 3)=e^{A_{2} \cdot(2 T / 3-d T)} \cdot x(d T-T / 3)+\int_{d T-T / 3}^{T / 3} e^{A_{2} \cdot(T / 3-\tau)} \cdot B_{2} u d \tau=\Phi_{2} \cdot x(d T-T / 3)+\text { Integ }_{2} .
$$

At switching surface $\Sigma_{2}$ for $t=d T$, the state vector can be expressed as

$$
x(d T)=e^{A_{3} \cdot(d T-T / 3)} \cdot x(T / 3)+\int_{T / 3}^{d T} e^{A_{3} \cdot(d T-\tau)} \cdot B_{3} u d \tau=\Phi_{3} \cdot x(T / 3)+\text { Integ }_{3} .
$$

For the second clock pulse $t=2 T / 3$, the state vector is

$$
x(2 T / 3)=e^{A_{4} \cdot(2 T / 3-d T)} \cdot x(d T)+\int_{d T}^{2 T / 3} e^{A_{4} \cdot(2 T / 3-\tau)} \cdot B_{4} u d \tau=\Phi_{4} \cdot x(d T)+\text { Integ }_{4} .
$$

The state vector at the switching surface $\Sigma_{3}$ is given by

$$
x(d T+T / 3)=e^{A_{5} \cdot(d T-T / 3)} \cdot x(2 T / 3)+\int_{2 T / 3}^{d T+T / 3} e^{A_{5} \cdot(d T+T / 3-\tau)} \cdot B_{5} u d \tau=\Phi_{5} \cdot x(2 T / 3)+\text { Integ }_{5} .
$$

At the end of the cycle $(t=T)$, the state evolves as

$$
x(T)=e^{A_{6} \cdot(2 T / 3-d T)} \cdot x(d T+T / 3)+\int_{d T+T / 3}^{T} e^{A_{6} \cdot(T-\tau)} \cdot B_{6} u d \tau=\Phi_{6} \cdot x(d T+T / 3)+\text { Integ }_{6} .
$$

Substituting the state variables $x(d T-T / 3)$ (Equation (41)) by Equation (40), $x(T / 3)$ (Equation (42)) by Equation (41), $x(d T)$, (Equation (43)) by Equation (42), $x(2 T / 3)$ (Equation (44)) by Equation (43), $x(d T+T / 3)$ (Equation (45)) by Equation (44) leads to the initial state vector $x(0)$, as follows:

$$
\begin{aligned}
& x(0)=\left(\mathbb{I}_{5}-\Phi_{6} \Phi_{5} \Phi_{4} \Phi_{3} \Phi_{2} \Phi_{1}\right)^{-1} \cdot\left(\Phi_{6} \Phi_{5} \Phi_{4} \Phi_{3} \Phi_{2} \cdot \text { Integ }_{1}+\Phi_{6} \Phi_{5} \Phi_{4} \Phi_{3} \cdot \text { Integ }{ }_{2}+\right. \\
& \left.+\Phi_{6} \Phi_{5} \Phi_{4} \cdot \text { Integ }_{3}+\Phi_{6} \Phi_{5} \cdot \text { Integ }_{4}+\Phi_{6} \cdot \text { Integ }_{5}+\text { Integ }_{6}\right) \text {. }
\end{aligned}
$$

With Equation (29), the three switching conditions at different time moments (inside the interval $[0, T)$ ) are identified as

$$
\begin{array}{r}
s w_{1}(x, d t-T / 3)=k_{L} \cdot x_{4}(d T-T / 3)-x_{5}(d T-T / 3)+k_{p} k_{c} \cdot x_{1}(d T-T / 3)-k_{p} \cdot V_{r e f}=0 \\
s w_{2}(x, d T)=k_{L} \cdot x_{2}(d T)-x_{5}(d T)+k_{p} k_{c} \cdot x_{1}(d T)-k_{p} \cdot V_{r e f}=0
\end{array}
$$


and

$$
s w_{3}(x, d T+T / 3)=k_{L} \cdot x_{3}(d T+T / 3)-x_{5}(d T+T / 3)+k_{p} k_{c} \cdot x_{1}(d T+T / 3)-k_{p} \cdot V_{r e f}=0 .
$$

From Equations (40) and (47), Equations (40)-(42) and (48), Equations (40)-(44) and (49) are deduced as follows:

$$
\left[\begin{array}{lllll}
k_{p} k_{c} & 0 & 0 & k_{L} & -1
\end{array}\right] \cdot \Phi_{1} \cdot x(0)+\text { Integ }_{1}-k_{p} \cdot V_{r e f}=0,
$$

$$
\begin{gathered}
{\left[\begin{array}{lllll}
k_{p} k_{c} & k_{L} & 0 & 0 & -1
\end{array}\right] \cdot\left(\Phi_{3} \Phi_{2} \Phi_{1} \cdot x(0)+\Phi_{3} \Phi_{2} \cdot \text { Integ }_{1}+\Phi_{3} \cdot \text { Integ }_{2}+\text { Integ }_{3}\right)-k_{p} \cdot V_{\text {ref }}=0} \\
\text { and }
\end{gathered}
$$

$$
\begin{aligned}
& {\left[\begin{array}{lllll}
k_{p} k_{c} & 0 & k_{L} & 0 & -1
\end{array}\right] \cdot\left(\Phi_{5} \Phi_{4} \Phi_{3} \Phi_{2} \Phi_{1} \cdot x(0)+\Phi_{5} \Phi_{4} \Phi_{3} \Phi_{2} \cdot \text { Integ }_{1}+\right.} \\
& \left.+\Phi_{5} \Phi_{4} \Phi_{3} \cdot \text { Integ }_{2}+\Phi_{5} \Phi_{4} \cdot \text { Integ }_{3}+\Phi_{5} \cdot \text { Integ }_{4}+\text { Integ }_{5}\right)-k_{p} \cdot V_{\text {ref }}=0 .
\end{aligned}
$$

Each integral $I n t_{i}$ and $\Phi_{i}(i=\overline{1,6})$ only depend on the $d$ duty cycle, as well as the initial state vector $x(0)$. After substituting $x(0)$ into Equations (50),(51), or (52), these equations can be solved numerically with the Newton-Raphson method to obtain the value of $d$ for the periodic orbit. Afterwards, the eigenvalues of the monodromy matrix (28) can be calculated.

\section{Simulation Results}

\subsection{System Simulation under Healthy Conditions}

The healthy conditions are considered when the $M O S_{1}, M O S_{2}$, and $M O S_{3}$ are faultfree, Equation, $h_{1}=h_{2}=h_{3}=1$. The stability can be deduced by the evaluation of the eigenvalues of the monodromy matrix $\Phi_{\text {cycle } 1}$ of Equation (28) as the load $R$ changes from $1.5 \Omega$ to $2.5 \Omega$. This matrix has one complex conjugate pair and three real eigenvalues, illustrated in Figure 6. Table 1 shows some values of the Floquet multipliers of the monodromy matrix $\Phi_{\text {cycle } 1}$ of Equation (28) under healthy conditions. One of the real values is close to unity. We observe in Figure 6 and Table 1 that the eigenvalues 4 and 5 , for $R=1.5 \Omega$, are a pair of complex conjugated eigenvalues outside the unit circle, implying that the system is unstable. When $R$ increases from $1.5 \Omega$ to $2.5 \Omega$, the eigenvalues 4 and 5 move outside of the unit circle and finally cross the unit circle towards each other. Crossing the unit circle, the system undergoes a bifurcation phenomenon, leading to the Neimark bifurcation. The inductor current $i_{L 1}=x_{2}$ is plotted in the left and the right panels of Figure 7. Indeed, it can be observed that $x_{2}$ oscillates at the switching frequency $1 / T=100 \mathrm{kHz}$ for $R=2.5 \Omega$. Decreasing $R$ to $1.7 \Omega$, the chaotic behavior can also be observed.

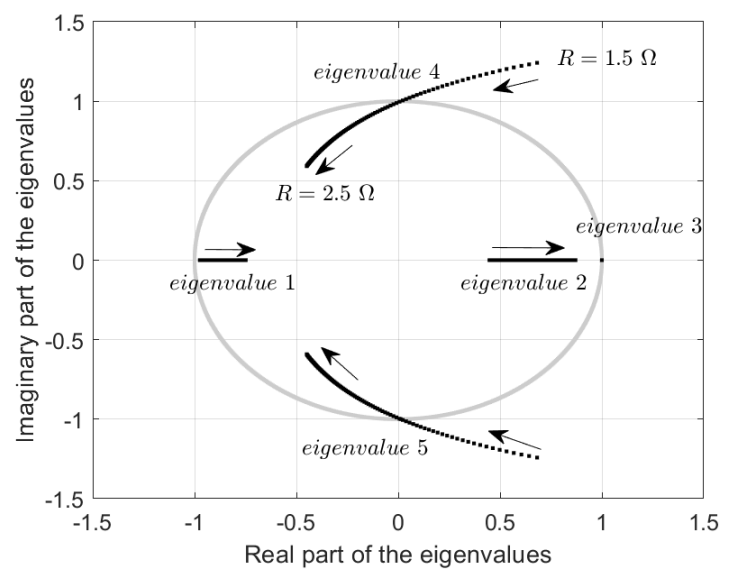

Figure 6. Locus of the eigenvalues of the monodromy matrix with $R$ variations under healthy conditions $h_{1}=h_{2}=h_{3}=1$. 
Table 1. Eigenvalues of the monodromy matrix as $R$ is varied in healthy switching case $h_{1}=h_{2}=h_{3}=1$.

\begin{tabular}{ccccc}
\hline $\mathbf{R}(\boldsymbol{\Omega})$ & Real Eigenvalue 1 & Real Eigenvalue 2 & Real Eigenvalue 3 & Complex Eigenvalues 45 \\
\hline 1.5 & -0.97455 & 0.44760 & 0.99957 & $0.68191 \pm i \cdot 1.24128$ \\
1.6 & -0.93415 & 0.56232 & 0.99955 & $0.34386 \pm i \cdot 1.14218$ \\
1.7 & -0.9002 & 0.6547 & 0.99954 & $0.11025 \pm i \cdot 1.04805$ \\
2 & -0.82493 & 0.79446 & 0.99949 & $-0.24993 \pm i \cdot 0.82523$ \\
2.2 & -0.78907 & 0.83561 & 0.99947 & $-0.36023 \pm i \cdot 0.71525$ \\
2.5 & -0.74851 & 0.87052 & 0.99944 & $-0.44962 \pm i \cdot 0.59171$ \\
\hline
\end{tabular}
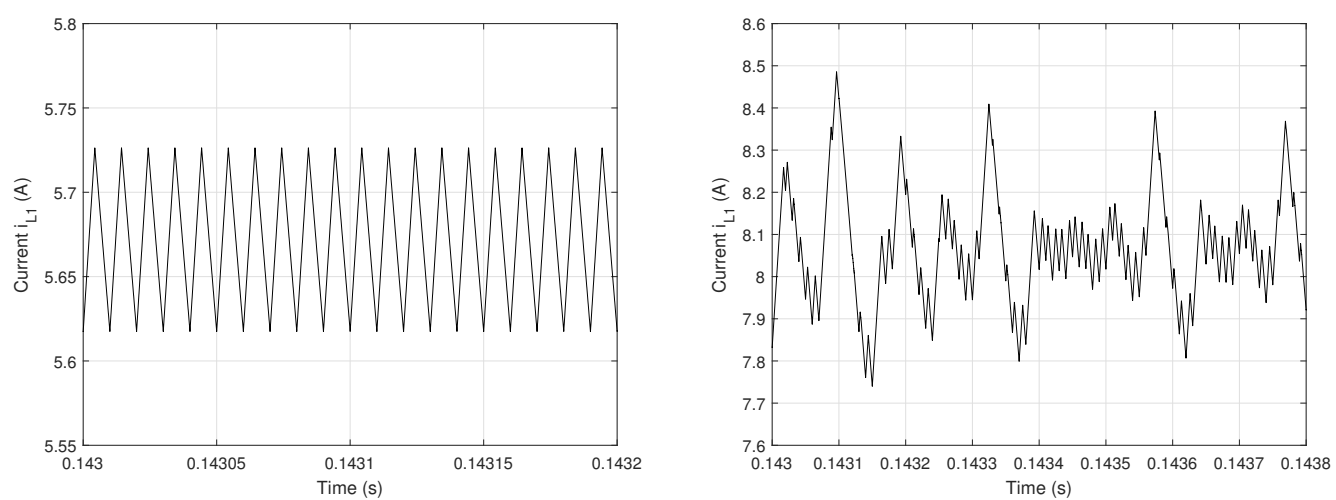

Figure 7. Time-domain waveforms for different values of $R$ (left panel $R=2.5 \Omega$, right panel $R=1.7 \Omega)$.

\subsection{System Simulation under One Open-Circuit Faulty Condition}

Let us consider that an open-circuit fault occurs in any MOS, e.g., in $\operatorname{MOS}_{3}\left(h_{3}=0\right)$. The following can be extended to the two others, $M O S_{1}$ or $M O S_{2}$, considering the symmetry in the interleaved boost converter. The analysis is similar wherever the fault is located. A high current flows through the components immediately after the fault occurrence. A too-slow circuit protection could cause a failure: the currents $i_{L 1}, i_{L 2}, i_{L 3}$ and the ripple of the output voltage $v_{\mathcal{C}}$ are affected. The current $i_{L 3}$ drops down to zero: this is why this state variable is outside of system analysis. Therefore, from now on, there are four state variables, $x_{1}, x_{2}, x_{3}$, and $x_{4}$ : the capacitor voltage $v_{c}$, the inductor currents $i_{L 1}, i_{L 2}$, and the output of the integrator $v_{i}$. As a consequence, the subtraction of the current $i_{L 3}$ (or using $h_{3}=0$ ) from the equations above, decreases the state vector from five to four elements. Inside the period $T$, there are four operation modes: ON OFF, ON ON, OFF ON, and OFF OFF, for the system under one open-circuit faulty condition. The evolution of the state variables in different mode during $[0, T)$ can be described by four matrices $A_{P}, A_{Q}, A_{R}, A_{S}(4 \times 4$ dimension) by subtracting the fourth column and line of the Matrices (7) or (10), (13), (16) or (19), (22). The matrices $A_{P}, A_{Q}, A_{R}$, and $A_{S}$ can be defined as

$$
A_{P}=\left[\begin{array}{cccc}
-\frac{1}{R C} & \frac{\bar{h}_{1}}{C} & \frac{1}{C} & 0 \\
-\frac{\bar{h}_{1}}{L} & 0 & 0 & 0 \\
-\frac{1}{L} & 0 & 0 & 0 \\
-k_{i} k_{c} & 0 & 0 & 0
\end{array}\right], \quad A_{Q}=\left[\begin{array}{cccc}
-\frac{1}{R C} & \frac{\bar{h}_{1}}{C} & \frac{\bar{h}_{2}}{C} & 0 \\
-\frac{\bar{h}_{1}}{L} & 0 & 0 & 0 \\
-\frac{\bar{h}_{2}}{L} & 0 & 0 & 0 \\
-k_{i} k_{c} & 0 & 0 & 0
\end{array}\right] \text {, }
$$

and 


$$
A_{R}=\left[\begin{array}{cccc}
-\frac{1}{R C} & \frac{1}{C} & \frac{\bar{h}_{2}}{C} & 0 \\
-\frac{1}{L} & 0 & 0 & 0 \\
-\frac{\bar{h}_{2}}{L} & 0 & 0 & 0 \\
-k_{i} k_{c} & 0 & 0 & 0
\end{array}\right], \quad A_{S}=\left[\begin{array}{cccc}
-\frac{1}{R C} & \frac{1}{C} & \frac{1}{C} & 0 \\
-\frac{1}{L} & 0 & 0 & 0 \\
-\frac{\bar{h}_{3}}{L} & 0 & 0 & 0 \\
-k_{i} k_{c} & 0 & 0 & 0
\end{array}\right] \text {. }
$$

In accordance with the diagram in Figure 8, the monotromy matrix

$$
\Phi_{\text {cycle } 2}=S_{S P} \cdot \Phi_{S}\left(T, \frac{T}{3}+d T\right) \cdot S_{R S} \cdot \Phi_{R}\left(\frac{T}{3}+d T, \frac{T}{3}\right) \cdot S_{Q R} \cdot \Phi_{Q}\left(\frac{T}{3}, d T\right) \cdot S_{P Q} \cdot \Phi_{P}(d T, 0),
$$

has four exponential transition matrices and four saltation matrices. The transition matrices $\Phi_{P}, \Phi_{Q}, \Phi_{R}$, and $\Phi_{S}$ depend on the matrices $A_{P}, A_{Q}, A_{R}$, and $A_{S}$. The saltation matrices have the following form:

$$
S_{P Q}=\left[\begin{array}{cccc}
1-\frac{\left(1-\bar{h}_{2}\right) \cdot x_{3} k_{p} k_{c}}{\operatorname{den}_{P Q} \cdot C} & -\frac{\left(1-\bar{h}_{2}\right) \cdot x_{3} k_{L}}{\operatorname{den}_{P Q} \cdot C} & 0 & \frac{\left(1-\bar{h}_{2}\right) \cdot x_{3}}{\operatorname{den}_{P Q} \cdot C} \\
0 & 1 & 0 & 0 \\
\frac{\left(1-\bar{h}_{2}\right) \cdot x_{1} k_{p} k_{c}}{\operatorname{den}_{P Q} \cdot L} & -\frac{\left(1-\bar{h}_{2}\right) \cdot x_{1} k_{L}}{\operatorname{den}_{P Q} \cdot L} & 1 & -\frac{\left(1-\bar{h}_{2}\right) \cdot x_{1}}{\operatorname{den}_{P Q} \cdot L} \\
0 & 0 & 0 & 1
\end{array}\right],
$$

with

$$
\operatorname{den}_{P Q}=\left(k_{i} k_{c}-\frac{k_{p} k_{c}}{R C}-\frac{k_{L} \bar{h}_{1}}{L}\right) \cdot x_{1}+k_{p} k_{c} \frac{\bar{h}_{1}}{C} \cdot x_{2}+\frac{k_{p} k_{c}}{C} \cdot x_{3}+\frac{k_{L}}{L} \cdot V_{i n}-k_{i} \cdot V_{\text {ref }} .
$$

and

$$
S_{R S}=\left[\begin{array}{clcc}
1+\frac{\left(1-\bar{h}_{2}\right) \cdot x_{3} k_{p} k_{c}}{d e n_{R S} \cdot C} & 0 & \frac{\left(1-\bar{h}_{2}\right) \cdot x_{3} k_{L}}{d e n_{R S} \cdot C} & -\frac{\left(1-\bar{h}_{2}\right) \cdot x_{3}}{d e n_{R S} \cdot C} \\
0 & 1 & 0 & 0 \\
-\frac{\left(1-\bar{h}_{2}\right) \cdot x_{1} k_{p} k_{c}}{d e n_{R S} \cdot L} & 0 & 1-\frac{\left(1-\bar{h}_{2}\right) \cdot x_{1} k_{L}}{\operatorname{den} R S} & \frac{\left(1-\bar{h}_{2}\right) \cdot x_{1}}{\operatorname{den}_{R S} \cdot L} \\
0 & 0 & 0 & 1
\end{array}\right],
$$

with

$$
d e n_{R S}=\left(k_{i} k_{c}-\frac{k_{p} k_{c}}{R C}-\frac{k_{L} \bar{h}_{2}}{L}\right) \cdot x_{1}+\frac{k_{p} k_{c}}{C} \cdot x_{2}+k_{p} k_{c} \frac{\bar{h}_{2}}{C} \cdot x_{3}+\frac{k_{L}}{L} \cdot V_{i n}-k_{i} \cdot V_{r e f} .
$$

where $S_{S P}=S_{Q R}=\mathbb{I}_{4}$.

Once these matrices are found, the four eigenvalues of the monodromy matrix $\Phi_{\text {cycle } 2}(55)$ are determined (Figure 9), where the right panel is an extension of the left panel. Special attention is paid to the movement of the Floquet multipliers. Three eigenvalues remain inside of the unit circle and one is outside: they are given in Table 2 for some values of $R$. It can be observed that the fourth eigenvalue of the monodromy matrix $\Phi_{\text {cycle } 2}$ of Equation (55) is always superior to one, indicating a sudden loss of stability for any value of $R$ inside the interval $[1.5 \Omega, 2.5 \Omega]$. 


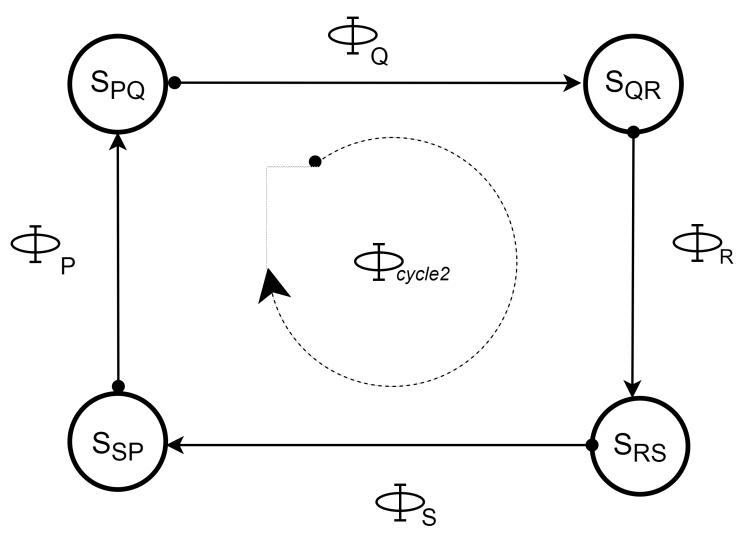

Figure 8. Diagram of the transition matrices and the saltation matrices in faulty switching case $h_{3}=0$, $h_{1}=h_{2}=1$.
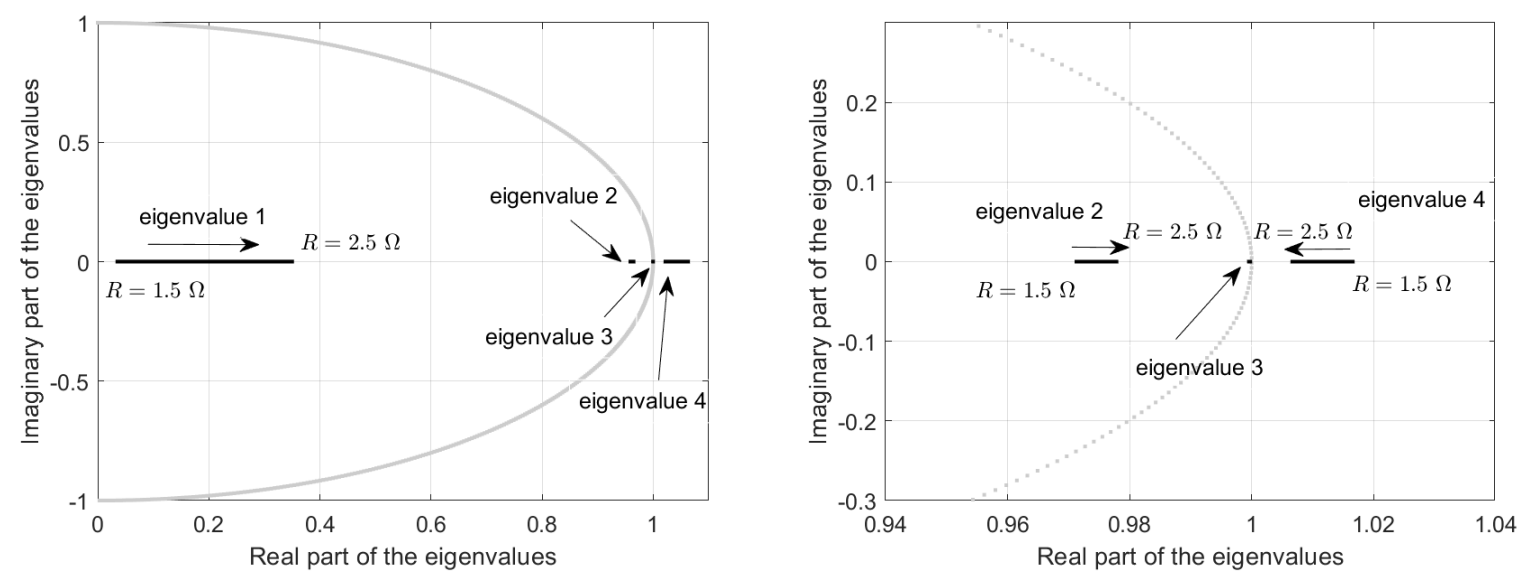

Figure 9. Locus of the eigenvalues of the monodromy matrix in faulty switching case $h_{3}=0$, $h_{1}=h_{2}=1$, when $R$ varies.
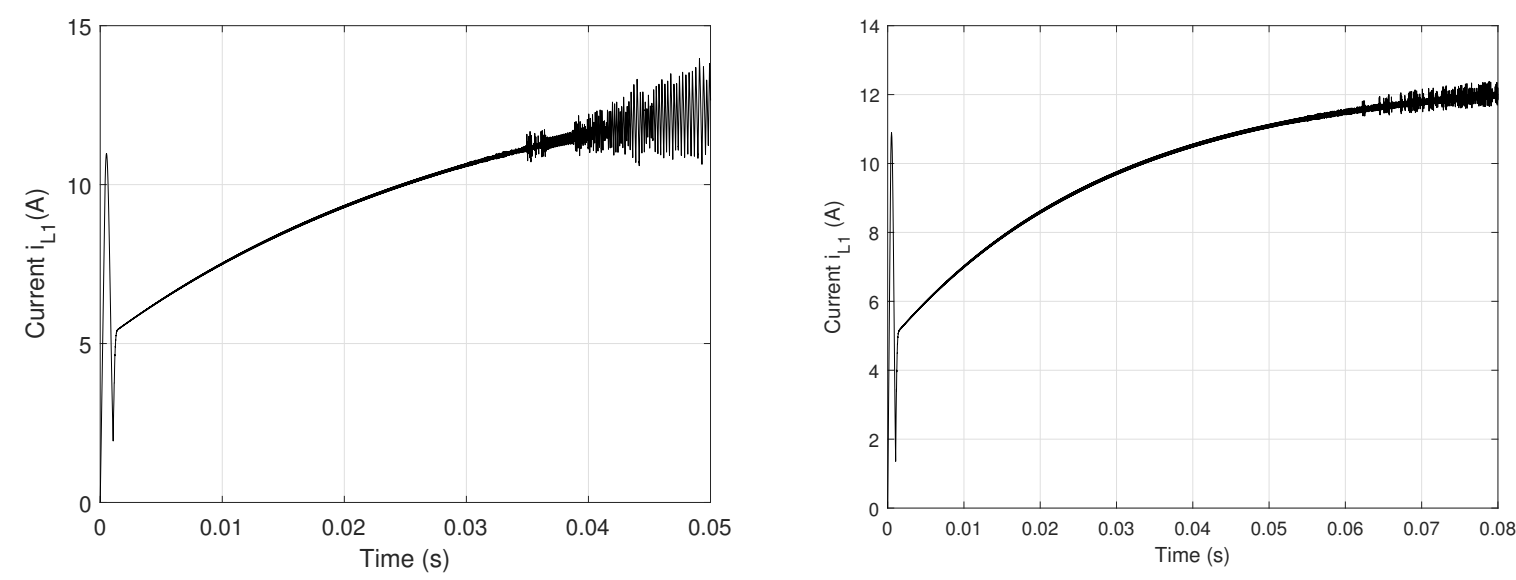

Figure 10. Time-domain waveforms for different values of $R$ (left panel $R=1.5 \Omega$, right panel $R=1.7 \Omega$ ) and in faulty switching case $h_{1}=h_{2}=1, h_{3}=0$. 


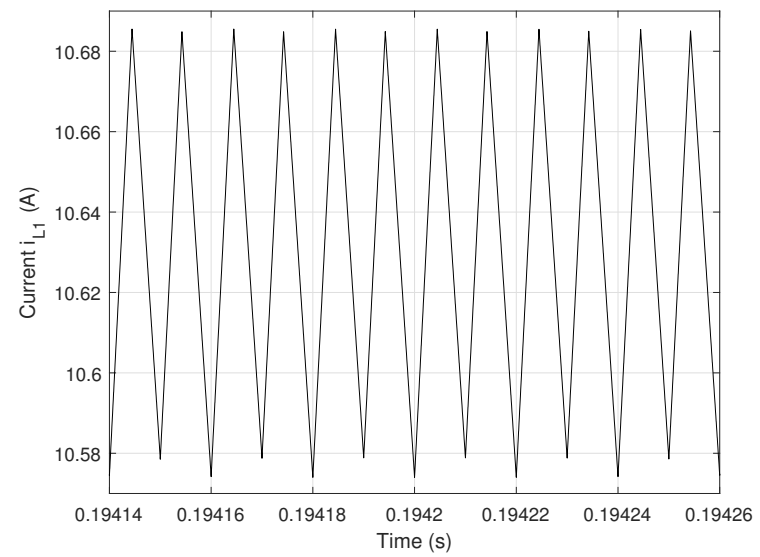

Figure 11. Time-domain waveform for $R=2 \Omega$ in faulty switching case $h_{1}=h_{2}=1, h_{3}=0$.

Table 2. Eigenvalues of the monodromy matrix in faulty switching case $h_{3}=0, h_{1}=h_{2}=1$, as $R$ varies.

\begin{tabular}{ccccc}
\hline $\mathbf{R}(\boldsymbol{\Omega})$ & Real Eigenvalue $\mathbf{1}$ & Real Eigenvalue 2 & Real Eigenvalue 3 & Real Eigenvalues 4 \\
\hline 1.5 & 0.862122 & 0.99999 & 0.999705 & 1.02199 \\
1.6 & 0.056013 & 0.96466 & 0.999701 & 1.02531 \\
1.7 & 0.079817 & 0.96455 & 0.999694 & 1.02867 \\
2 & 0.166681 & 0.96329 & 0.999678 & 1.03943 \\
2.2 & 0.234999 & 0.96187 & 0.999669 & 1.04763 \\
2.5 & 0.349913 & 0.95898 & 0.999657 & 1.06275 \\
\hline
\end{tabular}

When $R$ increases from $1.5 \Omega$ to $2.5 \Omega$, the eigenvalues 4 are always outside of the unit circle: the system is unstable. Indeed, it can be observed that the inductor current $i_{L 1}=x_{2}$ (plotted in the left panel of Figure 10 for $R=1.5 \Omega$ ) presents a no-periodicity undergoing to a chaotic behavior. It is essential to study the occurrence of chaos due to the variation of the system parameters. When a bifurcation occurs, an abrupt change in the steady-state behavior of the system also occurs. The very irregular form of $i_{L 1}$ is due to the intrinsic nonlinear dynamics driven by the on and off durations of the switch MOS which vary during a period of the clock. When $R$ increases to $1.7 \Omega$, the behavior remains chaotic, as shown in the right panel of Figure 10. Figure 11 finally represents the inductor current $i_{L 1}$ for $R$ to $2.2 \Omega$. This periodic waveform is also called period $-2 T$, because the pattern of a large peak followed by a small peak is repeated approximately once every two clock cycles.

\subsection{Development of a Fault-Tolerant Strategy with the Slope Compensation}

The same voltage and inner current controller are employed for the closed loops control of the converter. Based on $[26,27,35]$, it is possible to increase the stability with the movement of the eigenvalues from the outside to the inside of the unit circle, by changing the monodromy matrix $\Phi_{\text {cycle } 2}$ (55). The eigenvalues are outside of the unit circle in opencircuit faulty condition, which indicates instability. The monodromy $\Phi_{\text {cyclez }}$ matrix depends on $\Phi_{P}, \Phi_{Q}, \Phi_{R}$, and on $\Phi_{S}$, and the saltation matrices $S_{P Q}$ and $S_{R S}$. The exponential of matrices $\Phi_{P}, \Phi_{Q}, \Phi_{R}$, and $\Phi_{S}$ depend on the state matrices $A_{P}, A_{Q}, A_{R}$, and $A_{S}$; they cannot be modified because they are dependent on the design of the converter [26,27]. To guarantee the stability of 1T-periodic orbit, we propose to modify the saltation matrices $S_{P Q}$ and $S_{R S}$ and, consequently, the switching functions. These switching functions affect the saltation matrices $S_{P Q}$ and $S_{R S}$ by their time derivatives. Changing the switching function by addition of a slope compensation, the time derivative of the saltation matrices can be influenced, in agreement with the observations reported in earlier studies [26,27]. The vector norm of the saltation matrices $S_{P Q}$ and $S_{R S}$ is independent of state values, because the control feedback is based on the change of the ramp time signal limits. 
A fault-tolerant strategy with slope compensation is employed to avoid the instability illustrated in Figure 12. This strategy is applied to improve degraded mode operation and to allow continuity of functionality of the interleaved boost converter. The slope compensation requires few additional components (Figure 12) and has the following form:

$$
V_{\text {slope }}=V_{L}+a \cdot \frac{t}{T^{\prime}}
$$

where $a=V_{U}-V_{L}$. Inside the interval $[0, T)$ at $t=d T$ and $t=d T+T / 3$, two switching conditions exist and have the following forms:

$$
k_{p} k_{c} \cdot x_{1}(d T)-k_{p} \cdot V_{r e f}-x_{4}(d T)-k_{L} \cdot x_{2}(d T)+V_{L}+a \cdot d=0,
$$

and

$k_{p} k_{c} \cdot x_{1}(d T+T / 3)-k_{p} \cdot V_{r e f}-x_{4}(d T+T / 3)-k_{L} \cdot x_{3}(d T+T / 3)+\left(V_{L}+a \cdot d+\frac{a}{3}\right) \cdot e^{\frac{T}{3}}=0$.

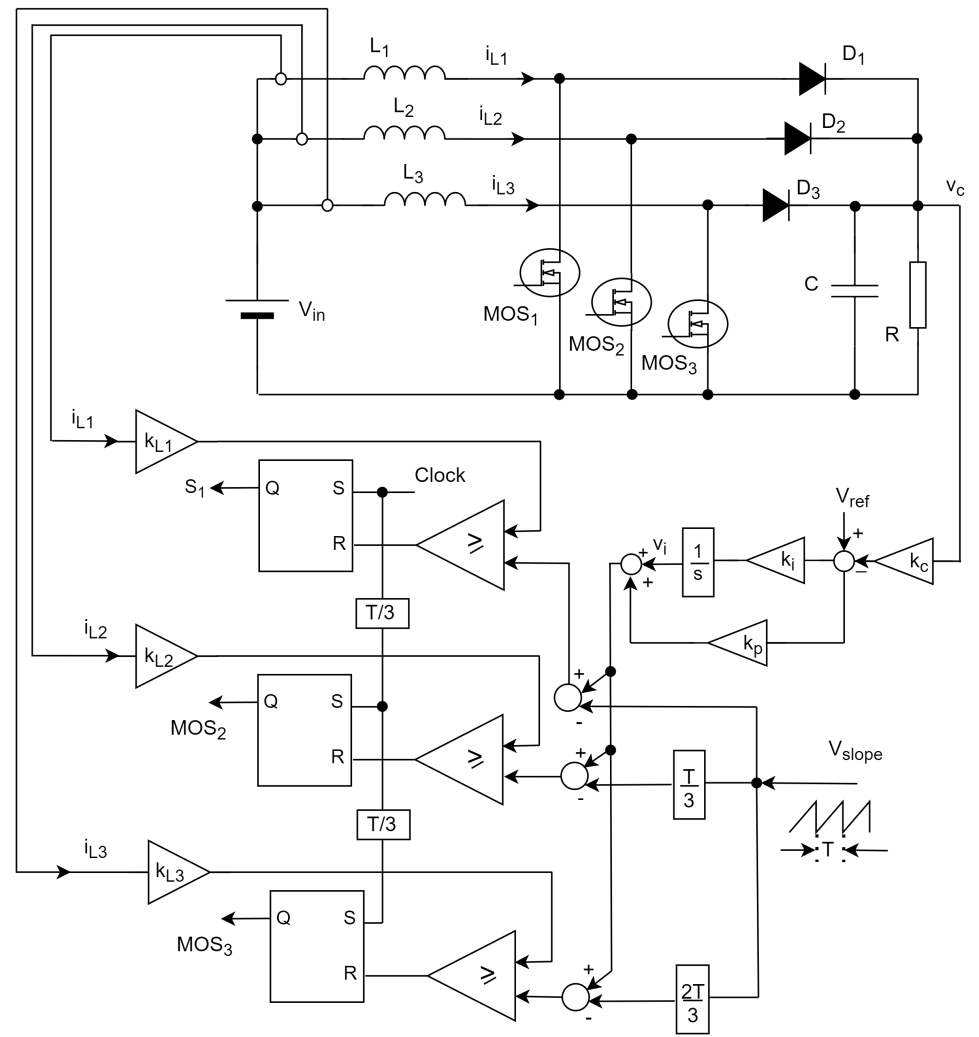

Figure 12. Interleaved boost converter with $V_{\text {in }}=14 \mathrm{~V}, V_{\text {ref }}=2.4 \mathrm{~V}, L_{1}=L_{2}=L_{3}=L=560 \mu \mathrm{H}, \mathrm{C}=$ $300 \mu \mathrm{F}, R=1.5 \Omega-2.5 \Omega, T=0.00001 \mathrm{~s}, k_{i}=50, k_{c}=0.1, k_{p}=0.5, k_{L 1}=k_{L 2}=k_{L 3}=k_{L}=0.1$.

The elements of the saltation matrices $S_{P Q}$ and $S_{R S}$ have the following denominators:

$$
\begin{gathered}
\operatorname{den}_{P Q}=\left(k_{i} k_{c}-\frac{k_{p} k_{c}}{R C}-\frac{k_{L} \bar{h}_{1}}{L}\right) \cdot x_{1}+k_{p} k_{c} \frac{\bar{h}_{1}}{C} \cdot x_{2}+\frac{k_{p} k_{c}}{C} \cdot x_{3}+\frac{k_{L}}{L} \cdot V_{i n}-k_{i} \cdot V_{\text {ref }}+\frac{a}{T} . \\
\operatorname{den}_{R S}=\left(k_{i} k_{c}-\frac{k_{p} k_{c}}{R C}-\frac{k_{L} \bar{h}_{2}}{L}\right) \cdot x_{1}+\frac{k_{p} k_{c}}{C} \cdot x_{2}+k_{p} k_{c} \frac{\bar{h}_{2}}{C} \cdot x_{3}+\frac{k_{L}}{L} \cdot V_{i n}-k_{i} \cdot V_{r e f}+\frac{a}{T} \cdot e^{\frac{T}{3}} .
\end{gathered}
$$

where $a / T$ and $(a / T) \cdot e^{T / 3}$ represent the time derivation of the surfaces.

Figure 13 shows the eigenvalues for several values of the load resistance $R$, indicating the stability of the interleaved boost converter. All the eigenvalues are inside the unit circle, as can be seen in Table 3 for certain values of $R$. 
The inductor current $x_{2}=i_{L 1}$ response with slope compensation for $R=1.5 \Omega$ and $R=2.5 \Omega$ is shown in Figure 14, respectively in Figure 15. It is clear that $x_{2}$ current, after a time transient (left panel of Figure 14, respectively left panel of Figure 15), settles down to the stable T-period limit cycle (right panel of Figure 14, respevtively right panel of Figure 15). A fault-tolerant strategy based on the slope compensation increases the stability of the interleaved boost converter under an open-circuit faulty condition within the whole range of the load $R=1.5-2.5 \Omega$. As its 1T-period orbit is stable, the eigenvalues of the monodromy matrix are all inside the unit circle.

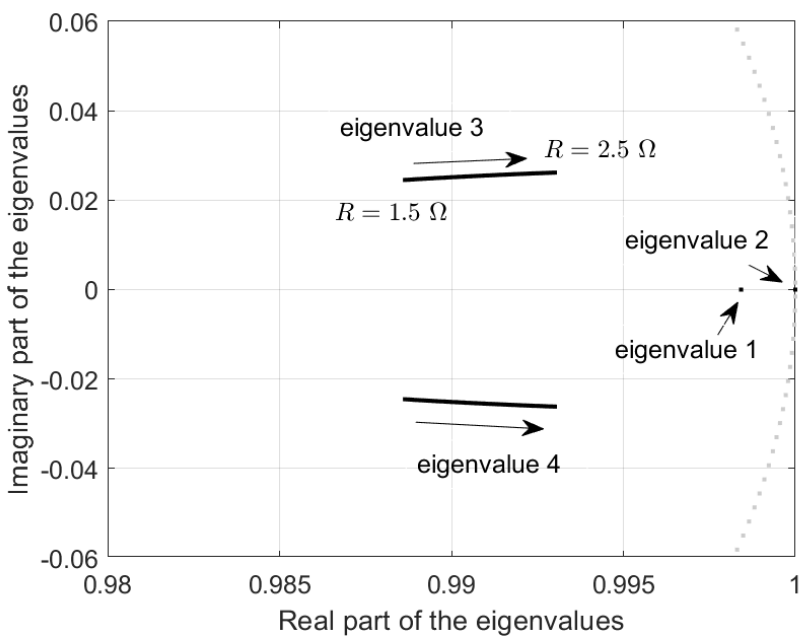

Figure 13. Locus of the eigenvalues of the monodromy matrix varying $R$ in faulty switching condition $h_{1}=h_{2}=1, h_{3}=0$, and with a slope compensation.

Table 3. Eigenvalues of the monodromy matrix in faulty switching case $h_{1}=h_{2}=1, h_{3}=0$ and with a slope compensation.

\begin{tabular}{cccc}
\hline $\mathbf{R}(\boldsymbol{\Omega})$ & Real Eigenvalue 1 & Real Eigenvalue 2 & Complex Eigenvalues 34 \\
\hline 1.5 & 0.984378 & 0.99999963 & $0.98864833 \pm i \cdot 0.02452323$ \\
1.6 & 0.984235 & 0.99999962 & $0.98932429 \pm i \cdot 0.02483646$ \\
1.7 & 0.984234 & 0.99999961 & $0.98992429 \pm i \cdot 0.02509463$ \\
2 & 0.984229 & 0.99999959 & $0.99136832 \pm i \cdot 0.02564743$ \\
2.2 & 0.984227 & 0.99999958 & $0.99211371 \pm i \cdot 0.02589673$ \\
2.5 & 0.984225 & 0.99999957 & $0.99300967 \pm i \cdot 0.02616516$ \\
\hline
\end{tabular}
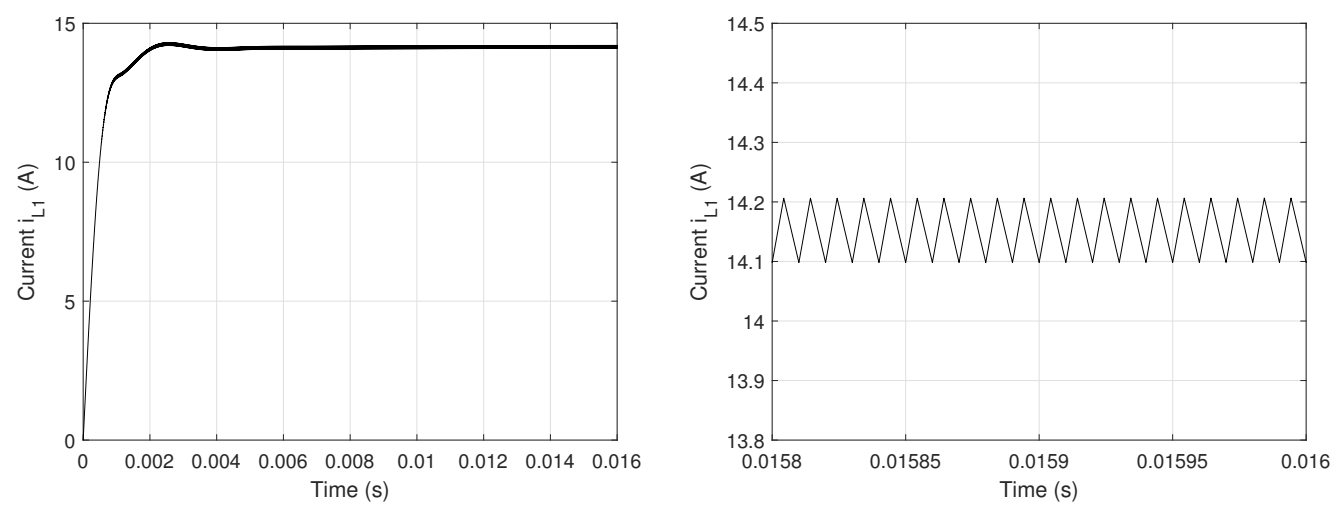

Figure 14. Time-domain waveforms for $R=1.5 \Omega$ in faulty switching case $h_{1}=h_{2}=1, h_{3}=0$, and with a slope compensation. 

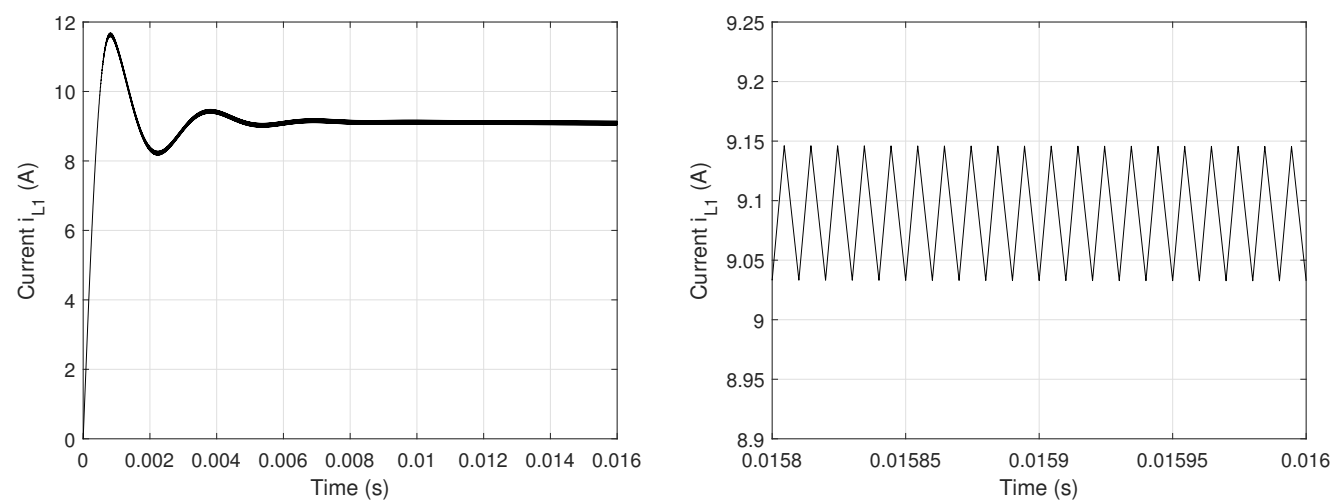

Figure 15. Time-domain waveforms for $R=2.5 \Omega$ in faulty switching case $h_{1}=h_{2}=1, h_{3}=0$, and with a slope compensation.

\section{Conclusions}

Interleaved converters use an increasing number of power electronics switches; this is an opportunity to develop fault-tolerant strategies. We herein introduced a novel mathematical function enabling to simultaneously model both states (Equation, healthy and faulty conditions) of an MOS switch, leading to a unique model of the system. It is noticeable that the proposed function is universally applicable to any interleaved or single-phase dc-dc converter. Then, we focused on the converter stability under healthy or faulty conditions with the monodromy matrix and its Floquet multipliers. They indicate a partial stability of the system with healthy conditions (for an interval of the load values) due to a Neimark bifurcation, and a total instability in open-circuit faulty conditions. Then, we introduced a fault-tolerant strategy based upon the peak current control with slope compensation: not only does it extend the stable range of the load variation, but it also ensures stability in faulty conditions, on the same extended interval. Finally, the simulation results validate the effectiveness of our method and confirm the theoretical analysis with emphasis on the open-circuit fault-tolerance capability. In addition, the suggested fault strategy can be extended to other power stages used in different applications.

Author Contributions: Formulation done by C.M. and A.A. Problem solved by C.M. All authors (C.M., A.A., R.S., T.A. and C.L.) contributed equally in numerical computations results discussion and writing manuscript. Revision done by C.M. All authors have read and agreed to the published version of the manuscript.

Funding: This research received no external funding.

Institutional Review Board Statement: Not applicable.

Informed Consent Statement: Not applicable.

Data Availability Statement: Not applicable.

Conflicts of Interest: The authors declare no conflicts of interest.

\section{References}

1. Aghdam, F.H.; Abapour, M. Reabilityand cost analysis of multistage boost converters connected to pv panels. IEEE J. Photovoltaics 2016, 6, 981-989. [CrossRef]

2. Ahmad, M.W.; Gorla, N.B.Y.; Malik H.; Panda S.K. A fault diagnosis and postfault reconguration scheme for interleaved boost converter in pv-based system. IEEE Trans. Power Electron 2021, 36, 3769-3780. [CrossRef]

3. Apablaza, D.; Munoz, J. Laboratory implementation of a boost interleaved converter for pv applications. IEEE Latin Am. 2016, 14, 2738-2743. [CrossRef]

4. Pei, T.; Hao, X. A fault detection method for photovoltaic systems based on voltage and current observation and evaluation. Energies 2019, 12, 1712. [CrossRef]

5. Guilbert, D.; N'Diaye, A.; Gaillard, A.; Djerdir, A. Fuel cell systems reliability and availability enhancement by developing a fast and efficient power switch open-circuit fault detection algorithm in interleaved dc-dc boost converter topologies. Int. J. Hydrogen Energy 2016, 41, 15505-15517. [CrossRef] 
6. Seyezhai, R.; Mathur, B.L. A comparison of three-phase uncoupled and directly coupled interleaved boost converter for fuel cell applications. Int. J. Electr. Eng. Informatics 2011, 3, 394-407. [CrossRef]

7. Marcos-Pastor, M.; Vidal-Idiarte, E.; Cid-Pastor, A.; Martinez-Salamero, L. Interleaved digital power factor correction based on the sliding-mode approach. IEEE Trans. Power Electron. 2016, 31, 4641-4653. [CrossRef]

8. Garcia, O.; Zumel, P.; de Castro, A.; Cobos, A. Automotive dc-dc bidirectional converter made with many interleaved buck stages. SIAM Rev. 2008, 50, 578-586. [CrossRef]

9. Garcia, O.; Zumel, P.; de Castro, A.; Cobos, A. An automotive 16 phases dc-dc converter. In Proceedings of the 2004 IEEE 35th Annual Power Electronics Specialists Conference (IEEE Cat. No.04CH37551), Aachen, Germany, 20-25 June 2004 ; pp. $350-355$.

10. Neugebauer, C.T.; Perreault, J. Computer aided optimisation of dc-dc converters for automotive applications. IEEE Trans. Power Electron. 2003, 18, 775-783. [CrossRef]

11. Banerjee, S.; Ghosh, A.; Rana, N. Design and fabrication of closed loop two-phase interleaved boost converter with type-iii controller. In Proceedings of the IECON - 42th Annual Conference of the IEEE Industrial Electronics Society, Florence, Italy, 23-26 October 2016; Volume 14, pp. 3331-3336.

12. Hui, L.; Ke, M.; Poh, L.; Frede, H. Online fault identification based on an adaptive observer for modular multilevel converters applied to wind power generation systems. Energies 2015, 8, 7140-7160.

13. Ni, L.; Patterson, D.J.; Hudgins, J.L. Maximum power extraction from a small wind turbine using a 4-phase interleaved boost converter. In Proceedings of the IEEE Power Electronics and Machines in Wind Applications, Lincoln, NE, USA, 2009; Volume 11, pp. 1-5.

14. Givi, H.; Farjah, E.; Ghanbari, T. Switch and diode fault diagnosis in nonisolated dc-dc converters using diode voltage signature. Int. J. Circuit Theory Appl. 2018, 65, 1606-1615. [CrossRef]

15. Pei, X.; Nie, S.Y.; Chen, Y.; Kang, Y. Open-circuit fault diagnosis and fault-tolerant strategies for full-bridge dc-dc converters. IEEE Trans. Power Electron. 2012, 27, 2550-2565. [CrossRef]

16. Shahbazi, M.; Jamshidpour, E.; Poure, P.; Saadate, S.; Zolghadri, M.R. Open- and short-circuit switch fault diagnosis for non isolated dc-dc converters using field programmable gate array. IEEE Trans. Ind. Electron 2013, 60, 4136-4146. [CrossRef]

17. Shahbazi, M.; Zolghadri, M.R.; Ouni, S.; Zolghadri, M.R. Fast and simple open-circuit fault detection method for interleaved dc-dc converters. In Proceedings of the 7th PEDSTC, Tehran, Iran, 16-18 February 2016; pp. 440-445.

18. Wassinger, N.; Penovi, E.; Retegui, R.G.; Maestri, S. Open-circuit fault identification method for interleaved converters based on time-domain analysis of the state observer residual. IEEE Trans. Power Electron 2019, 34, 3740-3749. [CrossRef]

19. Wei, H.; Zhang, Y.; Yu, L.; Zhang, M. A new diagnostic algorithm for multiple igbts open circuit faults by the phase currents for power inverter in electric vehicles. Energies 2018, 11, 1508. [CrossRef]

20. Zhuo, S.; Xu, L.; Gaillard, A.; Huangfu, Y.; Gao, F. Robust open-circuit fault diagnosis of multi-phase interleaved dc-dc boost converter based on sliding mode observer. IEEE Trans. Transp. Electrif 2019, 5, 638-649. [CrossRef]

21. Bento, F.; Marques Cardoso, A.J. A comprehensive survey on fault diagnosis and fault tolerance of dc-dc converters. Chin. J. Electron. 2018, 12, 1-12.

22. Li, P.; Li, X.; Zeng, T. A fast and simple fault diagnosis method for interleaved dc-dc converters based on output voltage analysis. Electronics 2021, 10, 1451. [CrossRef]

23. Zhang, Z.; Zhou, H.; Deng, C.; Song, Q. Multiloop interleaved control for three-level buck converter in solar charging applications. IEICE Electron. Express 2018, 15, 20180369. [CrossRef]

24. Banerjee, S.; Verghese, G.C. Nonlinear Phenomena in Power Electronics: Attractors, Bifurcations, Chaos, and Nonlinear Control; IEEE Press: New York, NY, USA, 2001.

25. Tse, C.K. Complex Behavior of Switching Power Converters; CRC Press: New York, NY, USA, 2003.

26. Giaouris, D.; Banerjee, S.; Zahawi, B.; Pickert, V. Stability analysis of the continuous-conduction-mode Buck converter via Filippovs method. IEEE Trans. Circuits Syst. 2008, 55, 1084-1096. [CrossRef]

27. Giaouris, D.; Maity, S.; Banerjee, S.; Pickert, V.; Zahawi, B. Application of Filippov's method for the analysis of subharmonic instability in dc-dc converters. Int. J. Circuit Theory Appl. 2009, 37, 899-919. [CrossRef]

28. Leine, R.I.; van Campen, D.H. Bifurcation phenomena in nonsmooth dynamical systems. Eur. J. Mech. Solids 2006, 25, 595-6168. [CrossRef]

29. Leine, R.I.; van Campen, D.H.; van de Vrande, B.L. Bifurcations in nonlinear discontinuous systems. Nonlinear Dyn. 2000, 23, 105-164. [CrossRef]

30. Morel, C.; Morel, J-Y.; Petreus, D. Generalization of the Filippov method for systems with a large periodic input. Math. Comput. Simul. 2018, 146, 1-17. [CrossRef]

31. di Bernardo, M.; Budd, C.J.; Champneys, A.R.; Kowalczyk, P.; Nordmark, A.B.; Tost, G.O.; Piiroinen, P.T. Bifurcations in nonsmooth dynamical systems. SIAM Rev. 2008, 50, 629-701. [CrossRef]

32. Kowalczyk, P.; di Bernardo, M. Two parameter degenerated sliding bifurcations in Filippov systems. Phys. D 2005, 20, 204-229. [CrossRef]

33. Kuznetsov, Y.A.; Rinaldi, S.; Gragnani, A. One-parameter bifurcations in planar Filippov systems. Int. J. Bifurc. Chaos 2003, 13, 2157-2188. [CrossRef]

34. Morel, C.; Petreus, D.; Rusu, A. Application of the Filippov method for the stability analysis of a photovoltaic system. Adv. Electr. Comput. Eng. 2011, 11, 93-98. [CrossRef] 
35. Wu, H.; Pickert, V.; Giaouris, D.; Ji, B. Nonlinear analysis and control of interleaved boost converter using real-time cycle to cycle variable slope compensation. IEEE Trans. Power Electron. 2017, 32, 7256-7270. [CrossRef]

36. Ott, E.; Grebogi, C.; Yorke, J.A. Controlling chaos. Phys. Rev. Lett. 1990, 64, 1196. [CrossRef]

37. Goharrizi, A.Y.; Khaki-Sedigh, A.; Sepehri, N. Observer-based adaptative control of chaos in nonlinear discret-time systems using time-delayed state feedback. Chaos Solitons Fractal 2009, 41, 2448-2455. [CrossRef]

38. Wang, N.; Lu, G.N.; Peng, X.Z.; Cai, Y.K. A research on time delay feedback control performance in chaotic system of dc-dc inverter. In Proceedings of the 2013 IEEE International Conference on Vehicular Electronics and Safety, Dongguan, China, 28-30 July 2013; pp. 215-218. 